\title{
Micromechanical modeling of the effect of elastic and plastic anisotropies on the mechanical behavior of $\beta$-Ti alloys
}

Safaa Lhadi $^{1,2}$, Stéphane Berbenni ${ }^{1,2 \star}$, Nathalie Gey ${ }^{1,2}$, Thiebaud Richeton ${ }^{1,2}$, Lionel Germain ${ }^{1,2}$

${ }^{1}$ Université de Lorraine, CNRS, Arts et Métiers ParisTech, LEM3, F-57000 Metz, France

${ }^{2}$ Laboratory of Excellence on Design of Alloy Metals for low-mAss Structures (DAMAS), Université de Lorraine, France

\section{Abstract}

Near $\beta$-titanium alloys like Ti-5553 or Ti-1023 often exhibit bimodal $\alpha$-phase constituents embedded in a retained $\beta$-phase matrix, which represents up to $40 \%$ of the volume. The highly elastic anisotropic $\beta$-phase may strongly influence the mechanical behavior of these alloys. The present work models the effect of the coupled role of $\beta$-phase elastic and plastic anisotropies on the local and overall responses of a fully $\beta$-phase polycrystalline aggregate like the Ti-17 alloy. The model is based on an advanced elasto-viscoplastic self-consistent (EVPSC) homogenization scheme solved by the "translated field" method together with an affine linearization of the viscoplastic flow rule. The effects of elastic anisotropy, crystallographic texture and grain morphology are theoretically studied during uniaxial tensile tests, tension-compression tests as well as multiaxial plastic yielding.

First, it is shown that different sets of elastic constants taken from literature give rise to similar effective responses but to widely scattered incompatibility stresses. During uniaxial tensile loading, the highest local incompatibility stresses are achieved in $<111>$ oriented grains at the end of the elastic regime. Likewise, the effect of the $\beta$-grain morphology for realistic grain aspect ratios is seen to be weak on the overall behavior but strong on incompatibility stresses. In addition, the elastic anisotropy can have a significant influence on yield surfaces for $\beta$-forged textured polycrystals. Finally, the simulated Bauschinger stress monotonically increases with the elastic anisotropy coefficient for a random texture while it may be reduced in case of $\beta$-forged texture due to a competition between elastic and plastic sources of incompatibility stresses.

Keywords: polycrystalline $\beta$-Ti; elastic anisotropy; elastic/plastic incompatibilities; Elasto-ViscoPlastic Self-Consistent (EVPSC) scheme; affine approximation; translated fields method *corresponding author, E-mail address: Stephane.Berbenni@univ-lorraine.fr 


\section{Introduction}

In the last decades, near- $\beta$ titanium alloys have benefit from a growing interest and are now competing with quasi- $\alpha$ or $\alpha / \beta$ titanium alloys for airframe forging applications like landing gears, turbine engines and rotor systems. Today, near- $\beta$ Ti-5553 and Ti-1023 alloys have become major candidates for landing gears under sever operating conditions (Nyakana et al., 2005). For these industrial alloys, high specific strength is achieved thanks to the building up of complex and multiscale $\alpha / \beta$ microstructures. A typical microstructure is schematized in Fig. 1 (Chini et al. (2016)). It is composed of hexagonal closed packed (HCP) a phase with two different morphologies (primary nodules and secondary platelets) embedded in a retained $\beta$ (BCC) matrix which represents up to $40 \%$ of the volume fraction. The HCP a phase is known to display plastic anisotropy (Cazacu et al., 2006; Khan et al., 2012; Meredith and Khan, 2012; Ghosh and Anahid, 2013). The $\beta$ phase is constituted of millimeter sized prior $\beta$-grains (approximately 1 to $2 \mathrm{~mm}$ long and $\sim 0.5 \mathrm{~mm}$ thick) fragmented into equiaxed $\beta$ sub-grains (5 to $10 \mu \mathrm{m}$ of diameter) of close orientations as shown at the meso-scale in Fig.1.

The mechanical behavior of these alloys is strongly dependent on their microstructures, especially on the nature and proportion of the phases, their morphologies and spatial distributions, their crystallographic texture and their chemical composition (Aeby-Gautier et al., 2011; Clément et al., 2007; Duval et al., 2011). The in-service microstructures often contain a large volume fraction of retained $\beta$-phase reported to exhibit high elastic anisotropy. However, the contribution of this elastically anisotropic $\beta$-phase on the mechanical behavior is still not well understood. A major difficulty relies on the determination of the true Single-crystal Elastic Constants (SEC) for the retained $\beta$ phase in the final microstructure. The published Single-crystal Elastic Constants (SEC) in the literature for $\beta$-Ti span over a large range of values (Fisher and Dever, 1970; Reid et al., 1973; Petry et al., 1991; Ledbetter et al., 2004; Fréour et al., 2005, 2011; Raghunathan et al., 2007; Tane et al., 2008; Kim and Rokhlin, 2009; Martin, 2012; Nejezchlebová et al., 2016; Hounkpati et al., 2016). In these studies, the Zener cubic anisotropy factor ranges from 1 to 8 . On the one hand, the SEC discrepancies found in the literature are due to the chemical composition of the $\beta$-phase in the different Ti-alloys studied by these authors. On the other hand, the SEC discrepancies come from the difficulties to measure the real SEC for near $\beta$-titanium alloys in the presence of $\alpha$-phase. First of all, ab initio calculations show the variation of the elastic properties of $\beta$-Ti alloys as a function of the chemical composition of the $\beta$-phase (Raabe et al., 2008) but these calculations give only the SEC at $0 \mathrm{~K}$, which does not correspond to the temperature range for the $\beta$-phase stability. Clément (2010) used tensile test to measure Young's modulus of Ti-LCB and Ti- 
5553 alloys with fully equiaxed $\beta$-phase microstructure and random texture. The Young's modulus of LCB ( 92GPa) was found much stiffer than the one of Ti-5553 alloy ( 68GPa). This is mainly due to the differences of the $\beta$-phase chemical composition. Nejezchlebová et al. (2016) measured with an ultrasonic technique the full tensor of elastic constants of the single crystal of Ti-LCB and found a Zener anisotropy coefficient of 2.37. Fréour et al. (2005) identified the SEC using X-ray diffraction measurements on a Ti-17 alloy and an inverse method based on a self-consistent model for elastic polycrystals (Hershey, 1954; Kröner, 1958). Later, a more refined self-consistent scheme was reported by Fréour et al. (2011) to account for needle-shaped a-phase grains elastically interacting with assumed spherical $\beta$-phase grains. Therefore, Fréour et al. $(2005,2011)$ reported two different sets of SEC whose anisotropy coefficients are 1.4 and 1.7, respectively. However, the inverse multiscale approach applied to multiphase polycrystalline alloys may give rise to multiple solutions. In addition, the micromechanical elastic strain concentration step contains approximations about the spatial distribution and morphologies of the phases.

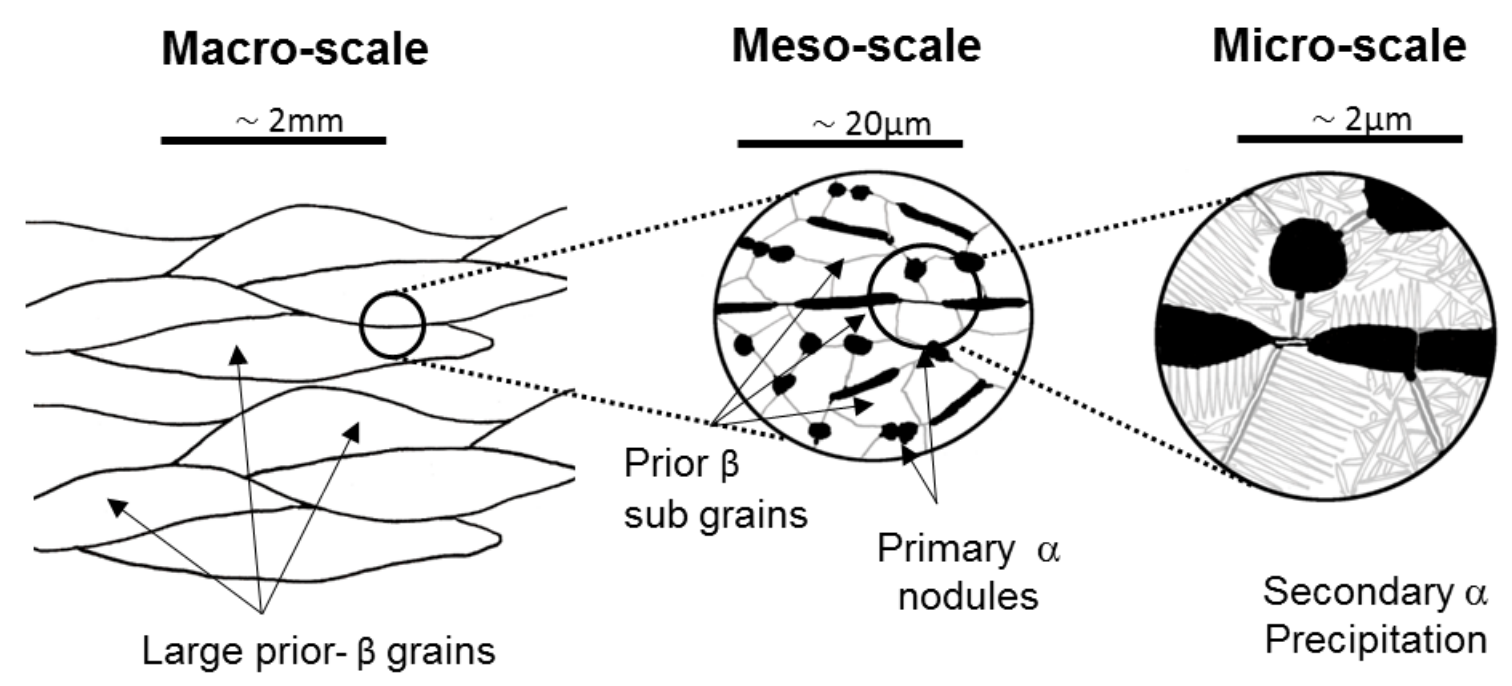

Figure 1- Schematization of the multiscale microstructure of near- $\beta$ Ti alloys (reproduced from Chini et al. 2016a).

The mechanical behavior of the $\beta$-phase may have a large influence on the elasto-viscoplastic behavior. Using crystal plasticity finite element modeling (CPFEM) with strain gradient plasticity, recent studies were performed on $\alpha / \beta$ titanium alloys such as forged Ti-6242 to study the effect of the $\beta$-phase in $\alpha / \beta$ colonies on dwell fatigue resistance (Anahid et al., 2012; Ashton et al., 2017). A viscoplastic self-consistent (VPSC) model was recently used by Mandal et al. (2017) to reproduce 
the stress/strain responses of Ti-5553 alloy based on uniaxial compression tests across a range of temperatures and strain rates. However, only a few studies aimed to describe the effect of $\beta$ phase elastic anisotropy in near $\beta$-titanium alloys on the local and overall elasto-(visco)plastic responses (Martin et al., 2011; Martin, 2012; Raghunathan et al., 2007; Hounkpati et al., 2016). In the case of Ti-1023 alloys, Raghunathan et al. (2007) applied a rate-independent EPSC (ElastoPlastic Self-Consistent) model (Turner and Tomé, 1994) in order to study the responses of an as forged and forged and aged Ti-1023 alloy. In particular, these authors used the EPSC model to fit the SEC of the $\beta$-phase by comparison with macroscopic curves and lattice strain measurements obtained from in situ synchrotron X-ray diffraction. Very different elastic constants were found in the as forged and in the aged alloys with Zener anisotropy coefficients of 1.9 and 8.3 respectively. This suggests that with enrichment in $\beta$-stabilizer, the $\beta$ elastic anisotropy increases in a huge range. In situ neutron measurements and modeling using an EPSC model of the inter-granular strains in the near-beta titanium alloy Ti- $\beta 21 \mathrm{~S}$ were recently performed by Hounkpati et al. (2016). Martin et al. (2011) studied the elasto-viscoplastic responses of Ti-5553 and Ti-17 alloys, using the classic Hill's (1965) interaction law in a rate-dependent form. In Martin (2012), an EVPSC model (Elasto-Visco-Plastic Self-consistent model) based on the "translated field" method earlier developed by Paquin et al. (1999, 2001), Sabar et al. (2002), Berbenni et al. (2004, 2007), Nicaise et al. (2011), was also employed for the same alloys to analyze the effect of different $\alpha / \beta$ volume fractions. The SEC of $\beta$-phase were chosen to fit the elastic stage of the tensile responses of Ti5553 alloy with $100 \% \quad \beta$-phase. The Zener anisotropy coefficient proper to his SEC is 2.4 . However, in these mentioned studies, the uncertainties of the $\beta$ SEC and the effect of its elastic anisotropy on the mechanical properties is not considered.

This contribution focuses on the role of the $\beta$-phase elastic anisotropy in the mechanical behavior of near- $\beta$ Ti alloys. The main objective is to theoretically investigate the coupled role of the $\beta$ phase elastic and plastic anisotropies on the elasto-viscoplastic local and overall responses of near $\beta$-titanium alloys with an advanced elasto-viscoplastic self-consistent scheme (EVPSC). The simulations are performed for a fully $\beta$-phase polycrystalline titanium aggregates and compared to the experimental tensile curves of Ti-5553 and Ti-17 provided by Settefrati (2012). The EVPSC model is applied to capture the elastic and plastic incompatibilities in $\beta$ alloys during tensile and tension-compression loadings for different $\beta$ grain morphologies and crystallographic textures. The proposed EVPSC scheme is based on the "translated field" method with affine linearization of the viscoplastic flow rule as recently developed by Mareau and Berbenni (2015). This advanced EVPSC scheme was successfully applied to pure $\alpha-T i$ to study its three-stage tensile hardening behavior (Amouzou et al., 2016). 
Hence, it is thought that the self-consistent approximation through an advanced EVPSC scheme is well suited to the modeling of disordered materials like fully $\beta$-phase titanium polycrystalline aggregates. While the original approaches of Paquin et al. (2001, 1999), Sabar et al. (2002), Berbenni et al. (2004) were derived from a classical secant approximation, this work adopts the first order affine linearization (Masson et al., 2000) of the viscoplastic flow rule, i.e. a first order Taylor expansion of the viscoplastic strain rate at the local scale. It has been observed that the first order affine formulation yields softer responses than the secant formulation (Masson et al., 2000; Masson and Zaoui, 1999; Molinari, 2001). Using the Mori-Tanaka scheme, a first order affine extension for elasto-viscoplastic two-phase composite materials was proposed by Berbenni and Capolungo (2015). In Mercier et al. (2012), it was shown that the predictions of the "translated field" method are very close to the ones obtained with the "additive interaction" law (Mercier and Molinari, 2009; Molinari, 2001). The "additive interaction law" was later adapted to build a finite strain elasto-viscoplastic self-consistent (EVPSC) model for polycrystals with "affine"-type approximation (Wang et al., 2010; Wang et al. 2013). In Berbenni and Capolungo (2015), good numerical results were obtained with the "translated field" method with affine linearization in comparison with the incremental variational approach developed by Lahellec and Suquet (2007) for two-phase fiber-reinforced composites. For polycrystals, incremental variational approaches were developed in the case of non-linear viscoplastic polycrystals (Liu et al., 2003). According to Berbenni and Capolungo (2015), Mareau and Berbenni (2015), the use of the first order affine formulation improves the estimate of the effective behavior which is in agreement with the full-field calculations (FEM, FFT), even when the viscoplastic flow rule is highly nonlinear.

The paper is organized as follows: Sections (2) and (3) are devoted to the description of the EVPSC model. In Section (2), the governing field equations of the elasto-viscoplastic problem are reviewed, and then the single crystal behavior with local viscoplastic flow rule for the $\beta$-phase is described. In Section (3), the "translated field" method to simplify the integral equation and the self-consistent approximation are described. Section (4) is dedicated to the selection of material parameters and discussion of the SEC considered in this study. Results and discussion are reported in Section (5). Numerical results account for the effects of the $\beta$-phase elastic anisotropy, the crystallographic texture and grain morphology during uniaxial tensile tests, cyclic loading as well as multiaxial plastic yielding. Finally, concluding remarks and prospects of this work are summarized in Section (6). 


\section{Constitutive equations and affine formulation}

In this paper, the scale transition from single- to poly-crystal is assessed through the EVPSC scheme first introduced in Mareau and Berbenni (2015) for thermo-elasto-viscoplastic heterogeneous materials. Here, thermal effects (i.e. thermal strains) are disregarded.

\subsection{Field equations}

In homogenization theory, the macroscopic stress rate and strain rate tensors $(\dot{\boldsymbol{\Sigma}}$ and $\dot{\mathbf{E}})$ of a Representative Volume Element (RVE) with volume V are obtained by volume averaging the local stress rate and strain rate tensors ( $\dot{\boldsymbol{\sigma}}$ and $\dot{\boldsymbol{\varepsilon}}$ ) as follows:

$\dot{\boldsymbol{\Sigma}}=\frac{1}{V} \int_{V} \dot{\boldsymbol{\sigma}}(\boldsymbol{x}) \boldsymbol{d} \boldsymbol{x}=\langle\dot{\boldsymbol{\sigma}}\rangle, \quad \dot{\boldsymbol{E}}=\frac{1}{V} \int_{V} \dot{\boldsymbol{\varepsilon}}(\boldsymbol{x}) \boldsymbol{d} \boldsymbol{x}=\langle\dot{\boldsymbol{\varepsilon}}\rangle$

Within the infinitesimal strain framework, the total local strain rate relative to an elasto-viscoplastic behavior (of Maxwell type) is decomposed into:

$\dot{\varepsilon}=\dot{\varepsilon}^{e}+\dot{\varepsilon}^{v p}$

with $\dot{\boldsymbol{\varepsilon}}^{\mathbf{e}}=\mathbf{s}$ : $\dot{\boldsymbol{\sigma}}$ from the generalized Hooke's law where $\mathbf{s}\left(=\mathbf{c}^{-1}\right)$ is the local elastic compliance tensor, and $\dot{\boldsymbol{\varepsilon}}^{\mathbf{v p}}=\mathbf{g}(\boldsymbol{\sigma})$ is the nonlinear viscoplastic constitutive law (plastic flow rule). Moreover, $\mathbf{g}(\boldsymbol{\sigma})$ is a nonlinear tensorial function dependent on the Cauchy stress $\boldsymbol{\sigma}$. In the absence of body force, the balance of linear momentum imposes the following stress and the stress rate equilibrium equations:

$\operatorname{div}(\sigma)=0$

$\operatorname{div}(\dot{\sigma})=0$

The kinematical compatibility condition for the total local strain rate yields:

$\dot{\varepsilon}=\nabla^{s} \dot{u}$

where $\dot{\mathbf{u}}$ is the velocity vector field and $\nabla^{\mathbf{s}}$ denotes the symmetric part of the gradient operator. On the boundary $\partial \mathrm{V}$ of $\mathrm{V}$, a homogeneous velocity vector $\dot{\mathbf{u}}^{\mathbf{d}}$ is imposed as follows:

$\dot{\boldsymbol{u}}^{\boldsymbol{d}}=\dot{\boldsymbol{E}} \cdot \boldsymbol{x}$ on $\partial V$

\subsection{Single crystal behavior}

The behavior of the $\beta$ single crystal is supposed to be elastic-viscoplastic where plastic distortion results from crystallographic slips on specific systems $s$ defined by a plane normal $n^{s}$ and a slip 
direction $\mathrm{l}^{\mathrm{s}}$. The slip systems considered for the BCC structure of the $\beta$ single crystal are specified in section 4.2. The phenomenological model with hardening variables developed by Méric et al. (1991); Méric and Cailletaud (1991) is used to describe the viscoplastic shear strain rate $\dot{\gamma}^{s}$ :

$\dot{\gamma}^{s}=\left\langle\frac{\left|\tau^{s}-x^{s}\right|-r^{s}}{K}\right\rangle^{n} \operatorname{sign}\left(\tau^{s}-x^{s}\right)$

where $\langle\mathrm{x}\rangle=\max (\mathrm{x}, 0)$ and $\tau^{\mathrm{s}}$ is the resolved shear stress, $\tau^{\mathrm{s}}=\mathbf{R}^{\mathrm{s}}: \boldsymbol{\sigma}$ with $\mathbf{R}^{\mathrm{s}}$ the symmetric Schmid

orientation tensor $\left(\mathrm{R}_{\mathrm{ij}}^{\mathrm{s}}=\frac{\mathrm{n}_{\mathrm{i}}^{\mathrm{S}} \mathrm{l}_{\mathrm{j}}^{\mathrm{s}}+\mathrm{n}_{\mathrm{j}}^{\mathrm{S}} \mathrm{l}_{\mathrm{i}}^{\mathrm{S}}}{2}\right)$. $\mathrm{x}^{\mathrm{s}}$ and $\mathrm{r}^{\mathrm{s}}$ are, respectively, the kinematic and the isotropic hardening shear stresses given by:

$x^{s}=c^{s} \alpha^{s} \quad$ with $\quad \dot{\alpha}^{s}=\dot{\gamma}^{s}-d^{s} \alpha^{s}\left|\dot{\gamma}^{s}\right|$

and

$r^{s}=r_{0}^{s}+\sum_{r=1}^{N} H_{s r} b^{r} Q^{r} q^{r} \quad$ with $\quad \dot{q}^{r}=\left(1-b^{r} q^{r}\right)\left|\dot{\gamma}^{r}\right|$

In the above equations, $\mathrm{K}$ and $\mathrm{n}$ are two material coefficients characterizing the viscous effect, $\mathrm{c}$, $\mathrm{b}, \mathrm{d}$ and $\mathrm{Q}$ are hardening parameters, $\mathbf{H}$ is an $\mathrm{N} \times \mathrm{N}$ matrix describing the interactions between the different slip systems, where $\mathrm{N}$ is the total number of the slip systems of the $\beta$ single crystal (here $\mathrm{N}=48$ for the BCC slip systems). Therefore, at the grain level, the viscoplastic strain rate is given by:

$\dot{\boldsymbol{\varepsilon}}^{v p}=\sum_{s=1}^{N} \boldsymbol{R}^{s}\left(\frac{\left|\tau^{s}-x^{s}\right|-r^{s}}{K}\right)^{n} \operatorname{sign}\left(\tau^{s}-x^{s}\right)$

\subsection{Affine formulation}

As mentioned previously, the viscoplastic strain rate is a nonlinear function of $\boldsymbol{\sigma}$. As in Mareau and Berbenni (2015), its affine linearization can be written as follows at a given reference stress:

$\dot{\varepsilon}^{v p}=g(\sigma) \approx m_{t}: \sigma+\dot{\eta}$

where $\mathbf{m}_{\mathbf{t}}$ (respectively $\mathbf{b}_{\mathbf{t}}=\mathbf{m}_{\mathbf{t}}{ }^{-1}$ ) is the tangent viscoplastic compliance tensor (respectively the tangent viscoplastic modulus tensor) defined by: 
$\boldsymbol{m}_{t}=\frac{\partial \boldsymbol{g}(\boldsymbol{\sigma})}{\partial \boldsymbol{\sigma}}$

$\dot{\eta}$ corresponds to the back-extrapolated viscoplastic strain rate tensor. Similar to the viscoplastic strain rate, the stress could be expressed as a nonlinear function $\mathbf{h}$ of $\dot{\boldsymbol{\varepsilon}}^{\mathbf{v p}}$ and then could be written in its linearized form (at a given reference viscoplastic strain) as:

$\sigma=h\left(\dot{\varepsilon}^{v p}\right) \approx b_{t}: \dot{\varepsilon}^{v p}+\zeta$

$\zeta$ is the back-extrapolated stress tensor related to the back-extrapolated viscoplastic strain rate by $\zeta=-\mathbf{b}_{\mathbf{t}}: \dot{\boldsymbol{\eta}}$. Therefore, the final linearized form of the constitutive law is:

$\dot{\varepsilon}=s: \dot{\sigma}+m_{t}: \sigma+\dot{\eta}$

According to the viscoplastic constitutive model used in this study (Eqs. (6-9)), $\mathbf{m}_{\mathbf{t}}=\partial \dot{\boldsymbol{\varepsilon}}^{\mathbf{v p}} / \partial \boldsymbol{\sigma}$ and $\dot{\eta}$ write in index notation, as

$m_{t, i j k l}=\sum_{s=1}^{N} \frac{n}{K}\left|\frac{\left|\tau^{s}-x^{s}\right|-r^{s}}{K}\right|^{n-1} R_{i j}^{s} R_{k l}^{s}$

$\dot{\eta}_{i j}=\sum_{s=1}^{N} R_{i j}^{s}\left(\left.\frac{\left|\tau^{s}-x^{s}\right|-r^{s}}{K}\right|^{n} \operatorname{sign}\left(\tau^{s}-x^{s}\right)-\sum_{s=1}^{N} \frac{n}{K}\left|\frac{\left|\tau^{s}-x^{s}\right|-r^{s}}{K}\right|^{n-1} R_{i j}^{s} \tau^{s}\right.$

\section{Scale transition: Elasto-Visco-Plastic Self-Consistent model (EVPSC) based on the Translated Field Method}

\subsection{Integral equation}

To recast the heterogeneous problem given by the assuming field equations in the form of an integral equation, a homogeneous reference medium -with uniform elastic moduli $\mathbf{C}$ (respectively, uniform elastic compliances $\mathbf{S}=\mathbf{C}^{-\mathbf{1}}$ ), uniform tangent viscoplastic moduli $\mathbf{B}_{\mathbf{t}}$ (respectively, uniform tangent viscoplastic compliances $\mathbf{M}_{\mathbf{t}}=\mathbf{B}_{\mathbf{t}}^{-\mathbf{1}}$ ) and uniform back-extrapolated strain rates $\dot{\mathbf{N}}$, is introduced. The local quantities $\mathbf{c}, \mathbf{s}, \mathbf{b}_{\mathbf{t}}, \mathbf{m}_{\mathbf{t}}$ and $\dot{\boldsymbol{\eta}}$ can then be expressed as the sum of a uniform reference quantity (respectively, $\mathbf{C}, \mathbf{S}, \mathbf{B}_{\mathbf{t}}, \mathbf{M}_{\mathbf{t}}$ and $\dot{\mathbf{N}}$ ) and a spatial fluctuating part (respectively, $\boldsymbol{\delta} \mathbf{c}, \boldsymbol{\delta} \mathbf{s}, \boldsymbol{\delta} \mathbf{b}_{\mathbf{t}}, \boldsymbol{\delta} \mathbf{m}_{\mathbf{t}}$ and $\left.\boldsymbol{\delta} \dot{\boldsymbol{\eta}}\right)$ as follows: 
$c(x)=C+\delta c(x), \quad s(x)=S+\delta s(x)$

$\mathbf{b}_{\mathrm{t}}=\mathbf{B}_{\mathrm{t}}+\delta \mathbf{b}_{\mathrm{t}}(\mathbf{x}), \quad \mathbf{m}_{\mathrm{t}}=\mathbf{M}_{\mathbf{t}}+\delta \mathbf{m}_{\mathrm{t}}(\mathbf{x})$

$\dot{\eta}(\mathbf{x})=\dot{\mathbf{N}}+\boldsymbol{\delta} \dot{\boldsymbol{\eta}}(\mathbf{x})$

Introducing both the elastic and tangent viscoplastic modified Green operators (see Kröner (1990)), denoted $\Gamma^{\mathbf{C}}$ and $\Gamma^{\mathbf{B}_{\mathbf{t}}}$ respectively, in combination with field equations and $1^{\text {st }}$ order affine formulation, the integral equation of the heterogeneous problem is obtained (Berbenni and Capolungo, 2015; Mareau and Berbenni, 2015):

$\dot{\varepsilon}=\dot{E}-\Gamma^{C} *\left(\delta c: \dot{\varepsilon}^{e}\right)-\Gamma^{B_{t}} *\left(\delta b_{t}: \dot{\varepsilon}^{v p}-b_{t}: \dot{\eta}\right)+\left(\Gamma^{C}: C-\Gamma^{B_{t}}: B_{t}\right) * \dot{\varepsilon}^{v p}$

where $*$ denotes the spatial convolution product. Note that the choice of the modified Green operators is due to their interesting properties for any compatible strain or strain rate field and any balanced stress or stress rate field -not detailed here- but for details, the reader is encouraged to refer back to the paper of Kröner (1990).

\subsection{Translated fields (TF) method}

The integral equation shows how the local strain rate depends on the macroscopic strain rate, the pure instantaneous elastic behavior, the pure time dependent viscoplastic behavior and the interactions between the two mechanisms. To deal with the differential nature (space-time coupling) of the integral equation -where analytical solutions do not exist, the translated fields (TF) method is introduced for heterogeneous nonlinear elasto-viscoplastic materials. The integral equation (Eq. 17) can be simplified using the solutions of the purely elastic and purely viscoplastic heterogeneous problem. So, the translated field decomposition applied to the total strain rate (Mareau and Berbenni, 2015; Paquin et al., 1999) writes:

$\dot{\varepsilon}=\dot{\boldsymbol{e}}^{\prime}+\dot{\boldsymbol{e}}^{\prime \prime}+\dot{\boldsymbol{\varepsilon}}^{*}$

where $\dot{\mathbf{e}}^{\prime}$ (resp. $\dot{\mathbf{e}}^{\prime \prime}$ ) are pure linear elastic (resp. pure nonlinear viscoplastic) compatible fields subjected to the same stress state as the elasto-viscoplastic heterogeneous material. $\dot{\mathbf{e}}^{\prime}$ is naturally chosen as the solution of the purely elastic heterogeneous problem, whereas $\dot{\mathbf{e}}^{\prime \prime}$ is naturally chosen as the solution of the purely viscoplastic heterogeneous problem, considered as a "thermo-elastic" linear comparison material (using the aforementioned affine extension). The solutions for the pure elastic problem and the pure viscoplastic problem with affine formulation are recalled in Appendix A, section (A.1). The last term $\dot{\boldsymbol{\varepsilon}}^{*}$ of the decomposition is the translated strain rate field which is a compatible field and represents the complexity of the space-time interactions 
due to the elastic and viscoplastic couplings. This one is associated to residual balanced stress (rate) fields $\dot{\boldsymbol{\sigma}}^{\prime}$ and $\boldsymbol{\sigma}^{\prime \prime}$ defined as:

$\dot{\varepsilon}^{*}=s: \dot{\sigma}^{\prime}+m_{t}: \sigma^{\prime \prime}$

From the definitions of compatible fields $\dot{\mathbf{e}}^{\prime}$ and $\dot{\mathbf{e}}^{\prime \prime}$, their solutions write:

$\dot{\boldsymbol{e}}^{\prime}=\left\langle\dot{\boldsymbol{\varepsilon}}^{e}\right\rangle-\Gamma^{C} *\left(\delta c: \dot{\boldsymbol{e}}^{\prime}\right)$

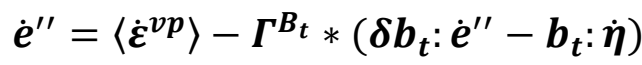

Based on the translated field properties and following equation (17), the associated integral equation is now considered:

$\dot{\varepsilon}^{*}=\dot{E}^{*}-\Gamma^{C} *\left(\delta c: s: \dot{\sigma}^{\prime}\right)-\Gamma^{B_{t}} *\left(\delta b_{t}: \boldsymbol{m}_{t}: \sigma^{\prime \prime}\right)+\left(\Gamma^{C}: C-\Gamma^{B_{t}}: B_{t}\right) *\left(\boldsymbol{m}_{t}: \sigma^{\prime \prime}\right)$

where $\dot{\mathbf{E}}^{*}$ is the macroscopic strain rate field from which boundary conditions are prescribed on $\delta \mathrm{V}$. Following Eq. (A13) in Appendix (A.1), we have $\dot{\mathbf{E}}^{*}=\left\langle\dot{\boldsymbol{\varepsilon}}^{*}\right\rangle=0$.

\subsection{Self-Consistent (SC) approximation}

In order to solve the TF problem given by Eqs. (20-22), the one-site self-consistent approximation is used. The main idea of the self-consistent model is to weaken the non-local parts of the modified Green operators -which are difficult to estimate-, that decrease as $1 /\left|\mathbf{x}-\mathbf{x}^{\prime}\right|^{3}$ (Kröner, 1990) by imposing averaging self-consistency conditions such that non-local interactions can be neglected. So for this reason, we introduce in the integral equations (20-22) the decomposition of the modified Green operator $\Gamma^{C}$ (respectively $\Gamma^{\boldsymbol{B}_{t}}$ ) associated with the elastic (respectively viscoplastic) reference homogeneous medium into a local part $\Gamma_{l}^{C}$ (respectively $\Gamma_{l}^{\boldsymbol{B}_{t}}$ ) and a nonlocal part $\boldsymbol{\Gamma}_{n l}^{C}$ (respectively $\boldsymbol{\Gamma}_{n l}^{B_{t}}$ ). Note that the decomposition of the modified Green operators is accompanied by the introduction of four unknown uniform tensors $\mathbf{Y}_{1}, \mathbf{Y}_{2}, \mathbf{Y}_{3}$ and $\mathbf{Y}_{4}$, which are chosen later to fulfill both the self-consistency conditions and the properties deduced from the averaging relations (Eq. 1) (see Appendix A.1). The explicit expressions of the integral equations (20-22) accounting for the decomposition of the modified Green operators into local and non-local parts, and the solutions of the four tensors $\mathbf{Y}_{1}, \mathbf{Y}_{2}, \mathbf{Y}_{3}$ and $\mathbf{Y}_{4}$ when neglecting the non-local terms 
are given in Appendix (A.2). Hence, the EVPSC solution of the associated problem is given by reducing the modified Green tensors to their local parts (Mareau and Berbenni, 2015):

$$
\begin{aligned}
\dot{e}^{\prime}=\left\langle\dot{\varepsilon}^{e}\right\rangle-\Gamma_{l}^{C}:\left(\delta c: \dot{e}^{\prime}\right) \\
\dot{e}^{\prime \prime}=\left\langle\dot{\varepsilon}^{v p}\right\rangle-\Gamma_{l}^{B_{t}}:\left(\delta b_{t}: \dot{e}^{\prime \prime}-b_{t}: \dot{\boldsymbol{\eta}}+B_{t}^{e}: \dot{N}^{e}\right) \\
\dot{\varepsilon}^{*}=\dot{E}^{*}-\Gamma_{l}^{C}:\left(\delta c: s: \dot{\sigma}^{\prime}-c: A^{C}: S^{e}:\left\langle\dot{\sigma}^{\prime}\right\rangle\right)-\Gamma_{l}^{B_{t}}:\left(\delta b_{t}: m_{t}: \sigma^{\prime \prime}-b_{t}: A^{B_{t}}: M_{t}^{e}:\left\langle\sigma^{\prime \prime}\right\rangle\right) \\
+\left(\Gamma_{l}^{C}: C-\Gamma_{l}^{B_{t}}: B_{t}\right):\left(m_{t}: \sigma^{\prime \prime}\right)
\end{aligned}
$$

where $\mathbf{A}^{\mathbf{C}}=\left(\mathbf{I}+\Gamma_{\mathbf{l}}^{\mathbf{C}}: \boldsymbol{\delta} \mathbf{c}\right)^{-\mathbf{1}}$ and $\mathbf{A}^{\mathbf{B}_{\mathbf{t}}}=\left(\mathbf{I}+\Gamma_{\mathbf{l}}^{\mathbf{B}_{\mathbf{t}}}: \boldsymbol{\delta} \mathbf{b}_{\mathbf{t}}\right)^{-\mathbf{1}}$ (with $\mathbf{I}$ the fourth order unit tensor) are the strain rate concentration tensors associated, respectively, with the elastic and viscoplastic reference media. The tensors $\mathbf{S}^{\mathbf{e}}$ (with $\mathbf{C}^{\mathbf{e}}=\mathbf{S}^{\mathbf{e}-\mathbf{1}}$ ), $\mathbf{M}_{\mathbf{t}}^{\mathbf{e}}\left(\right.$ with $\mathbf{B}_{\mathbf{t}}^{\mathbf{e}}=\mathbf{M}_{\mathbf{t}}^{\mathbf{e}^{-1}}$ ) and $\dot{\mathbf{N}}^{\mathbf{e}}$ are the effective elastic compliance tensor, the effective tangent viscoplastic compliance tensor and the effective back-extrapolated strain rate tensor, respectively. For the purely elastic and purely viscoplastic heterogeneous problem using the self-consistent approximation, we need to choose $\mathbf{C}($ resp. $\mathbf{S})=$ $\mathbf{C}^{\mathbf{e}}\left(\right.$ resp. $\left.\mathbf{S}^{\mathbf{e}}\right), \mathbf{B}_{\mathbf{t}}\left(\right.$ resp. $\left.\mathbf{M}_{\mathbf{t}}\right)=\mathbf{B}_{\mathbf{t}}^{\mathbf{e}}\left(\right.$ resp. $\left.\mathbf{M}_{\mathbf{t}}^{\mathbf{e}}\right)$ and $\dot{\mathbf{N}}=\dot{\mathbf{N}}^{e}$, to ensure that averaging relations are verified. Combining equations (23-25) and using the properties deduced from the averaging relations (see Appendix A), the strain rate concentration is obtained (see Eq. (65) in Mareau and Berbenni (2015) without thermal strains):

$$
\begin{aligned}
\dot{\varepsilon}=A^{C}: \dot{E}+A^{C}: & \Gamma_{l}^{B_{t}}:\left(b_{t}: \dot{\eta}+B_{t}^{e}: \dot{N}^{e}\right)+A^{C}: \Gamma_{l}^{C}:\left(\delta c: \dot{\varepsilon}^{v p}+c: A^{C}:\left(\left\langle\dot{\varepsilon}^{v p}\right\rangle-\dot{E}^{v p, e}\right)\right) \\
& -A^{C}: \Gamma_{l}^{B_{t}}:\left(\delta b_{t}: \dot{\varepsilon}^{v p}-b_{t}: A^{B_{t}}:\left(M_{t}^{e}: \Sigma+\dot{N}^{e}-\left\langle\dot{\varepsilon}^{v p}\right\rangle\right)\right) \\
& +A^{C}:\left(\Gamma_{l}^{C}: C^{e}-\Gamma_{l}^{B_{t}}: B_{t}^{e}\right):\left(\dot{\varepsilon}^{v p}-A^{B_{t}}:\left\langle\dot{\varepsilon}^{v p}\right\rangle\right) \\
& -A^{C}:\left(\Gamma_{l}^{C}: C^{e}-\Gamma_{l}^{B_{t}}: B_{t}^{e}\right):\left(A^{B_{t}}: \Gamma_{l}^{B_{t}}:\left(b_{t}: \dot{\eta}-B_{t}^{e}: \dot{N}^{e}\right)\right)
\end{aligned}
$$

Substituting the strain rate Eq. (26) in the local constitutive law (Eq. 13) gives the stress rate concentration equation, which is consistent with Eq. (66) in Mareau and Berbenni (2015) without thermal strains: 


$$
\begin{aligned}
\dot{\sigma}=c: A^{C}:\left(S^{e}:\right. & \left.\dot{\Sigma}+\dot{E}^{v p, e}\right)-c: \dot{\varepsilon}^{v p}+c: A^{C}: \Gamma_{l}^{B_{t}}:\left(b_{t}: \dot{\eta}+B_{t}^{e}: \dot{N}^{e}\right) \\
& +c: A^{C}: \Gamma_{l}^{C}:\left(\delta c: \dot{\varepsilon}^{v p}+c: A^{C}:\left(\left\langle\dot{\varepsilon}^{v p}\right\rangle-\dot{E}^{v p, e}\right)\right) \\
& -c: A^{C}: \Gamma_{l}^{B_{t}}:\left(\delta b_{t}: \dot{\varepsilon}^{v p}-b_{t}: A^{B_{t}}:\left(M_{t}^{e}: \Sigma+\dot{N}^{e}-\left\langle\dot{\varepsilon}^{v p}\right\rangle\right)\right) \\
& +c: A^{C}:\left(\Gamma_{l}^{C}: C^{e}-\Gamma_{l}^{B_{t}}: B_{t}^{e}\right):\left(\dot{\varepsilon}^{v p}-A^{B_{t}}:\left\langle\dot{\varepsilon}^{v p}\right\rangle\right) \\
& -c: A^{C}:\left(\Gamma_{l}^{C}: C^{e}-\Gamma_{l}^{B_{t}}: B_{t}^{e}\right):\left(A^{B_{t}}: \Gamma_{l}^{B_{t}}:\left(b_{t}: \dot{\eta}-B_{t}^{e}: \dot{N}^{e}\right)\right)
\end{aligned}
$$

where the expressions of the effective viscoplastic strain rate $\dot{\mathbf{E}}^{\mathbf{v p}, \mathbf{e}}$, which is chosen to impose the condition $(\langle\dot{\boldsymbol{\sigma}}\rangle=\dot{\boldsymbol{\Sigma}})$ ), and the effective back-extrapolated strain rate $\dot{\mathbf{N}}^{e}$ are recalled in the section (A.3) of Appendix A, see Eqs. (A26-A29). In Eqs. $(26,27)$, the Eshelby tensors associated to $\mathbf{C}^{\mathbf{e}}$ and $\mathbf{B}_{\mathbf{t}}^{\mathbf{e}}$ are given by $\Gamma_{\mathbf{l}}^{\mathbf{C}}: \mathbf{C}^{\mathbf{e}}, \boldsymbol{\Gamma}_{1}^{\mathbf{B}_{\mathbf{t}}}: \mathbf{B}_{\mathbf{t}}^{\mathbf{e}}$ respectively.

\section{Material parameters}

\subsection{Choice of the Single-crystal Elastic Constants (SEC) of the $\beta$-Ti phase}

As seen in the introduction, the SEC of the $\beta$-phase reported in the literature for near- $\beta$ Ti alloys are widely different from one set to another. We give in Table (1) a selection of the three independent cubic elastic constants $\left(\mathrm{C}_{11}, \mathrm{C}_{12}, \mathrm{C}_{44}\right)$. These SEC are classified by increasing anisotropy coefficient $(A)$ as defined by Zener:

$A=\frac{2 C_{44}}{C_{11}-C_{12}}$

As one can see, the anisotropy coefficient is widely different from one set to another. Note that these differences are related to the chemical composition of the $\beta$ phase present in the tested alloys but originate also from the measurement methods. The effective Young's modulus of the $\beta$ phase with random texture and equiaxed grains was computed for each set of SEC (see Table 1) and compared to the one measured on a quenched Ti-5553 alloy to retain $100 \%$ of $\beta$ phase (Clément, 2010; Settefrati, 2012 - no presence of $\omega$ phase was reported by the authors). The effective Young's modulus was computed using the elastic self-consistent solution (Hershey, 1954; Kröner, 1958), for which an analytic expression exists (deWit, 2008) in case of an isotropic distribution of crystallographic orientations.

It is found that the Young moduli computed using the SEC given by Martin (2012), Petry et al. (1991) and Raghunathan et al. (2007) are close to the measured modulus for Ti-5553 alloy, which is about 68GPa. We highlight that these three sets give very different Zener anisotropy coefficients with respective values of 2.4, 3 and 8.3. Therefore, the corresponding single crystal Young's 
moduli are different from each other's (see Fig. 2). As expected, the gap between the directional Young's moduli of single crystals along the directions $<100\rangle$ and $<111\rangle$ increases with the anisotropy coefficient A (see blue curve in Fig. 2).

Since the three sets of SEC given in Martin (2012), Petry et al. (1991) and Raghunathan et al. (2007) successfully reproduce the effective Young's modulus of $100 \% \beta$ Ti-5553 alloy, we keep them all in the next part of the paper to study the effect of the elastic anisotropy of the $\beta$-phase on the elasto-viscoplastic behavior of this alloy. 


\begin{tabular}{|c|c|c|c|c|c|c|}
\hline \multirow[b]{2}{*}{ References } & \multirow[b]{2}{*}{ Ti-based alloy } & \multicolumn{3}{|c|}{ SEC (GPa) } & \multirow[b]{2}{*}{$\mathbf{A}$} & \multirow{2}{*}{$\begin{array}{c}\text { Effective } \\
\text { Young's } \\
\text { modulus (GPa) } \\
\text { of } 100 \% \beta \\
\text { Computed in } \\
\text { this study } \\
\end{array}$} \\
\hline & & $\mathrm{C}_{11}$ & $\mathrm{C}_{12}$ & $\mathrm{C}_{44}$ & & \\
\hline Fréour et al. (2005) & $\mathrm{Ti}-17$ & 174 & 116 & 41 & 1.4 & 98.6 \\
\hline Fréour et al. (2011) & Ti-17 & 167 & 115 & 44 & 1.7 & 98.4 \\
\hline $\begin{array}{c}\text { Nejezchlebova et al. } \\
\qquad(2016)\end{array}$ & LCB & 138 & 102.2 & 42.5 & 2.37 & 83.8 \\
\hline Martin( 2012) & $\begin{array}{c}\text { Ti-17 } \\
\text { Ti-5553 }\end{array}$ & 100 & 70 & 36 & 2.4 & 69.4 \\
\hline Petry et al. (1991) & Pure & 134 & 110 & 36 & 3 & 66.6 \\
\hline Brandes et al. (1992) & Pure & 134 & 110 & 55 & 4.6 & 85.8 \\
\hline Fisher \& Dever (1970) & Pure & 99 & 85 & 33.6 & 4.8 & 52.8 \\
\hline Kim et al. (2009) & Ti-6242 & 135 & 113 & 54.9 & 5 & 83.7 \\
\hline Ledbetter et al. (2004) & Pure & 97.7 & 82.7 & 37.5 & 5 & 57.5 \\
\hline $\begin{array}{l}\text { Raghunathan et al. } \\
\qquad(2007)\end{array}$ & Ti-1023 & 140 & 128 & 50 & 8.3 & 67.2 \\
\hline
\end{tabular}

Table 1- A selection from the literature of Single-crystal Elastic Constants (SEC) for the $\beta$-phase. The values are classified by increasing Zener anisotropy coefficient given by $A=2 C_{44} /\left(C_{11}-C_{12}\right)$. For each SEC, the effective Young's modulus is computed with the elastic self-consistent solution (Hershey, 1954; Kröner, 1958) for a $100 \% \beta$ Ti alloy assuming equiaxed grains and random crystallographic texture. 


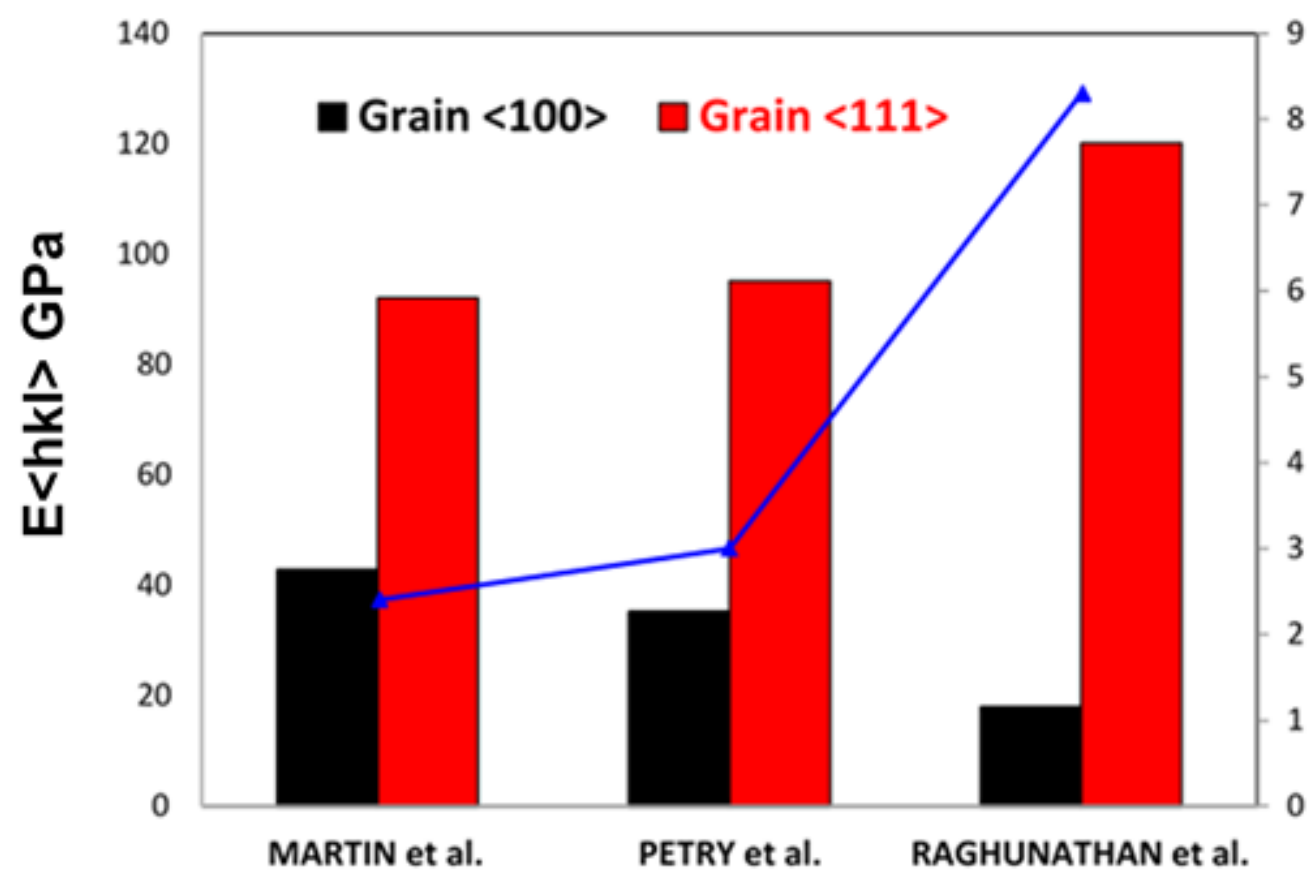

A

Figure 2- Directional Young's modulus of a single crystal for tensile direction parallel to crystallographic $<100\rangle$ (minimum) and $<111\rangle$ (maximum) directions obtained using the three selected $\beta$-phase SEC. The corresponding Zener anisotropy coefficient $(A)$ is also reported (blue curve).

\section{2. $\quad$ Single crystal plasticity parameters}

The $\beta$ single crystal plasticity parameters of the constitutive law of Méric et al. (1991) as introduced in section 2.2 were already studied and discussed by Martin et al. $(2011,2012)$ on Ti$5553100 \% \beta$ and $\mathrm{Ti}-17100 \% \beta$. Here, the elastic parameters corresponding to $A=3$ are used to identify the material parameters reported in Table 2 and associated with the viscoplastic flow rule and the strain-hardening model to be in good agreement with the experimental result (see Fig. 3). 


\begin{tabular}{|c|c|c|c|c|}
\cline { 2 - 5 } \multicolumn{1}{c|}{} & $\mathrm{n}$ & $\mathrm{K}\left(\mathrm{MPa} . \mathrm{s}^{1 / \mathrm{n}}\right)$ & $\mathrm{r}_{0}(\mathrm{MPa})$ & $\mathrm{c}(\mathrm{MPa})$ \\
\hline $\begin{array}{c}\{110\}<111> \\
12 \text { slip systems }\end{array}$ & 20 & 300 & 113 & 200 \\
\hline $\begin{array}{c}\{112\}<111> \\
12 \text { slip systems }\end{array}$ & 20 & 300 & 113 & 200 \\
\hline $\begin{array}{c}\{123\}<111> \\
24 \text { slip systems }\end{array}$ & 20 & 300 & 123 & 400 \\
\hline
\end{tabular}

Table 2- Viscoplastic flow rule and hardening parameters of the $\beta$-phase for each slip system family (Martin, 2012).

The procedure to identify the hardening and viscoplastic parameters is obtained from two tensile stress/strain curves at two different strain rates $\left(2 \times 10^{-4} s^{-1}\right.$ and $\left.2 \times 10^{-3} s^{-1}\right)$ for the $100 \% \beta$ Ti17 as reported on Fig. 3. First of all, the initial critical resolved shear stresses (CRSS) for the three slip system families denoted $r_{0}$ are reported in Table 2. The experimental yield strengths and the elastic-viscoplastic transition on the tensile stress-strain curves at both applied strain rates are used to find the best values for $r_{0}$ in the crystal plasticity model. The best fitted value for the CRSS associated with both $\{110\}<111>$ and $\{112\}<111>$ slip systems (i.e. the major active slip modes) was found to be $r_{0}=113 \mathrm{MPa}$ while the CRSS for $\{123\}<111>$ slip mode is a bit higher $\left(r_{0}=123 \mathrm{MPa}\right)$ in agreement with Martin et al. (2011,2012). It is noteworthy that Raghunathan et al. (2007) and Hounkpati et al. (2016) also discussed the CRSS values in the $\beta$-phase from in-situ synchrotron and neutron diffraction measurements respectively. Their numerical values were different in magnitude because these authors used a different crystal plasticity model and rate-independent EPSC schemes, but the CRSS for $\{110\}<111>$ and $\{112\}<111>$ slip systems were also found to be lower than the one for the $\{123\}<111>$ slip system. Furthermore, it was shown that a linear intragranular kinematic hardening model is sufficient to model the small hardening rate for such tensile monotonic responses then $\mathrm{c} \neq 0$ and $\mathrm{d}=0$ in Eq. (7). No isotropic hardening (and hardening matrix) was needed to make the hardening model more complex since both linear intra-granular kinematic hardening and inter-granular hardening originating from grain to grain interactions (Eq. (27)) were sufficient to well reproduce the overall hardening response up to $4 \%$ strain. As in the previous numerical study performed by Martin et al. (2011, 2012), a linear kinematic hardening at the scale of slip system is chosen with a c parameter for each slip system (Eq. (7)) identified from the stress/strain curve after the yield point. The $\mathrm{c}$ parameter was identified as $\mathrm{c}=200 \mathrm{MPa}$ for the $\{110\}<111>$ and the $\{112\}<111>$ slip systems and twice higher for $\{123\}<111>(\mathrm{C}=400 \mathrm{MPa})$, which is consistent with a low slip activity for this mode due to higher evolving flow strength with plastic 
deformation. After an overall strain of $4 \%$ at room temperature, it was observed that the $\beta$ phase transforms into the $\alpha$ " phase through precipitation (Aeby-Gautier et al., 2011; Settefrati, 2012). Therefore, a phase transformation induced hardening is present in the $\beta$ phase, which is out of the scope of the present model so that the parameters were identified on the tensile stress/strain curves up to $4 \%$ strain (see Fig. 3). Lastly, both material parameters $n$ and $K$ (see Table 2) describe the strain rate sensitive behavior of the material. They were identified to reproduce the low strain rate sensitive behavior between $1 \%$ and $4 \%$ strains using the experimental points in Fig. 3. Then, the increase of flow stress between both tensile responses at strain rates $2 \times 10^{-4} s^{-1}$ and $2 \times 10^{-3} s^{-1}$ is fully consistent with the available experimental data for $\mathrm{n}=20$ and $\mathrm{K}=300 \mathrm{MPa} \cdot \mathrm{s}^{1 / n}$.

\subsection{Polycrystal description}

A fully $\beta$-phase microstructure with either (i) a random crystallographic texture (i.e. an isotropic grain orientation distribution) or (ii) an anisotropic one composed of $60 \%$ of $<111>$ and $40 \%<100>$ fibers parallel to the loading direction (with a maximal disorientation of $5^{\circ}$ in both cases) is considered. This latter is typical of a $\beta$-forged material (Chaussy and Driver, 1996). In the following figures, we refer to the random and $\beta$-forged textures by the "No texture" and "With texture" terminologies, respectively. After forging, the $\beta$ grains shapes are ellipsoidal with different aspect ratios. In order to account for the grain shape effect on the overall behavior, grains are considered to be either spheres, oblate ellipsoids $(\mathrm{a}=\mathrm{b}>>\mathrm{c})$ or prolate ellipsoids $(\mathrm{a}=\mathrm{b}<<\mathrm{c}), \mathrm{a}, \mathrm{b}$ and $\mathrm{c}$ are the half-axes of the ellipsoids. In the following figures, when not specified, the grains are supposed to be spherical. In the model, the Eshelby tensors (Eshelby, 1957) associated with anisotropic effective moduli tensors $\mathrm{C}^{\mathrm{e}}, \mathrm{B}_{\mathrm{t}}^{\mathrm{e}}$ and the different ellipsoidal morphologies have been numerically computed using Gauss-Legendre numerical integration.

\section{Results and discussion}

Numerical results focus on the effect of the $\beta$-phase elastic anisotropy on the elasto-viscoplastic behavior with a special attention to the elastic-viscoplastic transition and to the development of incompatibility stresses. Several main classes of results are presented here considering the three selected sets of SEC.

First, the following simulations are made assuming spherical grains:

- Macroscopic stress-strain tensile test curves predicted for a random texture, compared to experimental data at two strain rates.

- Macroscopic stress-strain tensile test curves predicted for $\beta$-forged texture. 
Then, for both the random and $\beta$-forged textures,

- Incompatibility stresses are plotted for representative $<111>$ and $<100>$ grains oriented parallel to the loading direction,

- Yield surfaces obtained with different multiaxial loadings and,

- Strain-controlled cyclic stress-strain curves up to an applied strain amplitude of $5 \%$.

Finally, the effect of the $\beta$ grain ellipsoidal shape on the elastoviscoplastic behavior and on the incompatibility stresses is shown.

\subsection{Influence of elastic anisotropy on macroscopic tensile elasto-viscoplastic responses}

\subsubsection{Equiaxed $\beta$ microstructure with random texture}

Fig. 3 compares the tensile stress-strain curves obtained on Ti-17 (Settefrati, 2012) and the present model using the three selected sets of SEC and crystal plasticity parameters identified using SEC with $A=3$. For comparison, we plot in the same figure the stress-strain curves obtained for the elastic isotropic case $A=1$. As the texture is random, the computed macroscopic elastic tensor is isotropic. The considered Young's modulus and Poisson ratio are 69.4GPa and 0.35, respectively. In all figures, we refer to each set of SEC by its own anisotropy coefficient $A$ (see Table 1).

The three plotted stress-strain curves that use elastic anisotropy $A=2.4,3$ and 8.3 predict all the macroscopic elasto-viscoplastic response as well as the elastic-viscoplastic transitions for both strain rates $2 \times 10^{-3} s^{-1}$ and $2 \times 10^{-4} s^{-1}$ compared to the experimental data. For $A=1$, the predicted transition from the elastic domain to the viscoplastic one starts for much smaller stresses. For the four A values, the elastic-viscoplastic transitions are gradual because the texture is random. As expected by the self-consistent model, the stress-strain curves achieve the same viscoplastic asymptotic behavior when elastic strains become negligible (i.e. at large strains). However, the viscoplastic asymptotic state for $A=1$ is reached for higher strain, showing a longer transition between elastic and viscoplastic states contrary to the anisotropic cases $(A \neq 1)$. The asymptotic state is only reached at macroscopic strains of $\sim 15 \%$ and at $\sim 10 \%$ when strain rates are equal to $2 \times 10^{-3} s^{-1}$ and to $2 \times 10^{-4} s^{-1}$, respectively. 

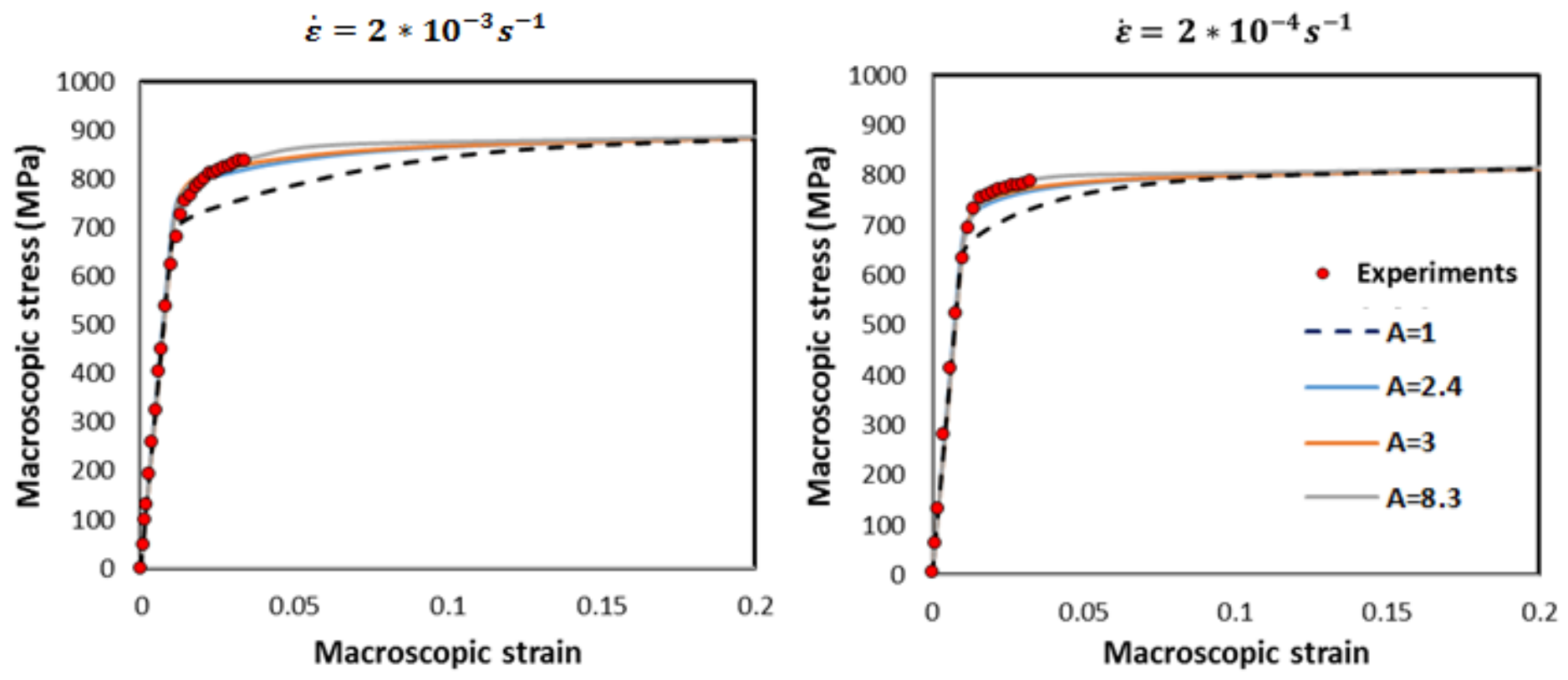

Figure 3- Tensile macroscopic stress $\left(\Sigma_{33}\right)$ vs macroscopic strain $\left(E_{33}\right)$ estimated by the Affine EVPSC model at two strain rates: $2 \times 10^{-3} s^{-1}$ and $2 \times 10^{-4} s^{-1}$ using four anisotropy factors $(\mathrm{A})$. The numerical results are compared to the experiments of Settefrati (2012) (red circles) (equiaxed $\beta$ microstructure with random texture).

\subsubsection{Equiaxed $\beta$ microstructure with $\beta$-forged texture}

The theoretical tensile stress-strain curves predicted by the present model for the $\beta$-forged texture (see Fig. 4) are plotted in Fig. 5 for the three anisotropy coefficients $A=2.4,3$ and 8.3. Again $A=1$ is added for comparison. The effective elastic moduli obtained using these anisotropy coefficients are similar. Unlike the results obtained for the random texture, the elastic-viscoplastic transition is sharper due to the nature of the texture characterized by $\langle 100\rangle$ and $\langle 111\rangle$ fibers aligned to the loading direction. The peculiar transition of the curve obtained with $A=8.3$ arises from the big differences of local behaviors (elastic and plastic incompatibilities) between $\langle 100\rangle$ and $\langle 111\rangle$ grains, due to the strong elastic anisotropy. These incompatibilities are explained in details in the following sections.

For $A=1$, the viscoplastic asymptotic state is reached earlier (macroscopic strains of $\sim 3.2 \%$ and $\sim 2.5 \%$ when strain rates are equal to $2 \times 10^{-3} \mathrm{~s}^{-1}$ and to $2 \times 10^{-4} \mathrm{~s}^{-1}$, respectively) compared to the random texture. This is mainly due to the strong plastic anisotropy induced by the $\beta$-forged texture. 

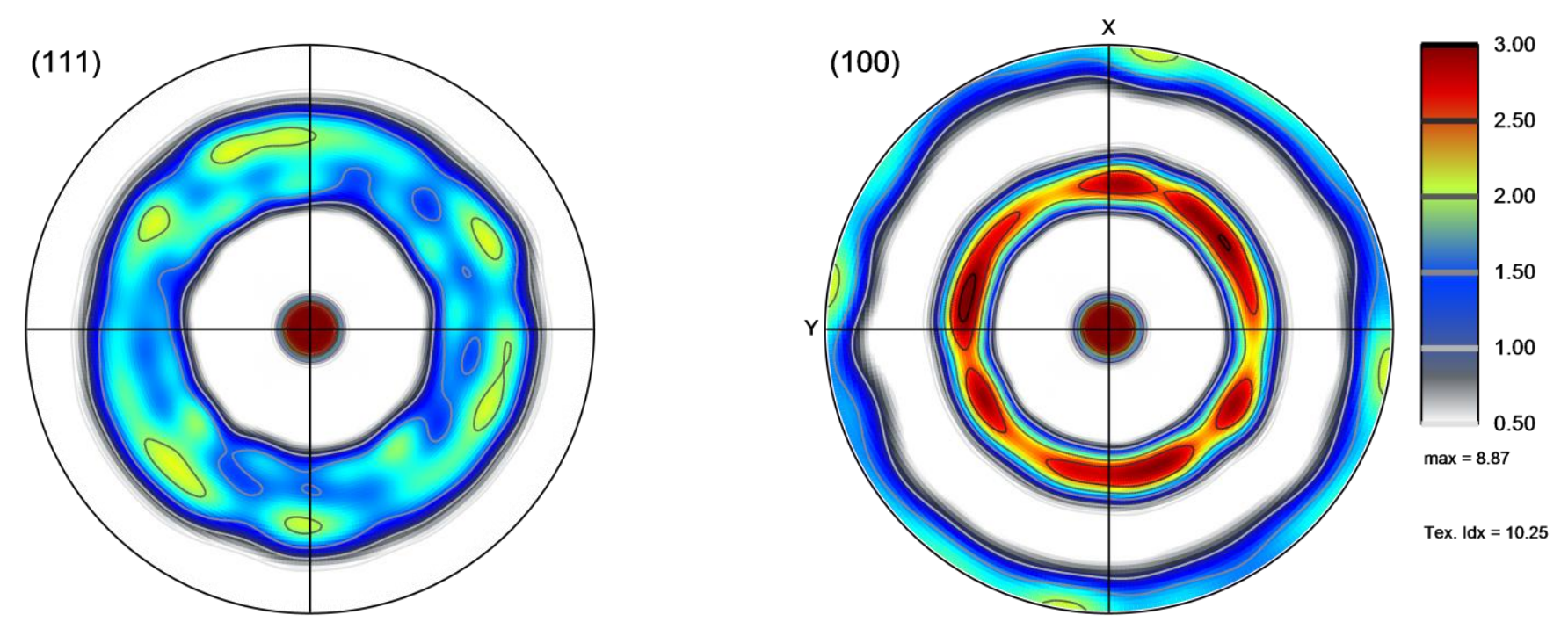

Figure 4- Stereographic (111) and (100) pole figures illustrating the $\beta$-forged crystallographic texture. The Texture Index measuring the degree of anisotropy of the texture (Bunge, 1982) is 10.25. These pole figures were generated using the ATEX-software (Beausir and Fundenberger, 2018).

$$
\dot{\varepsilon}=2 * 10^{-3} s^{-1}
$$

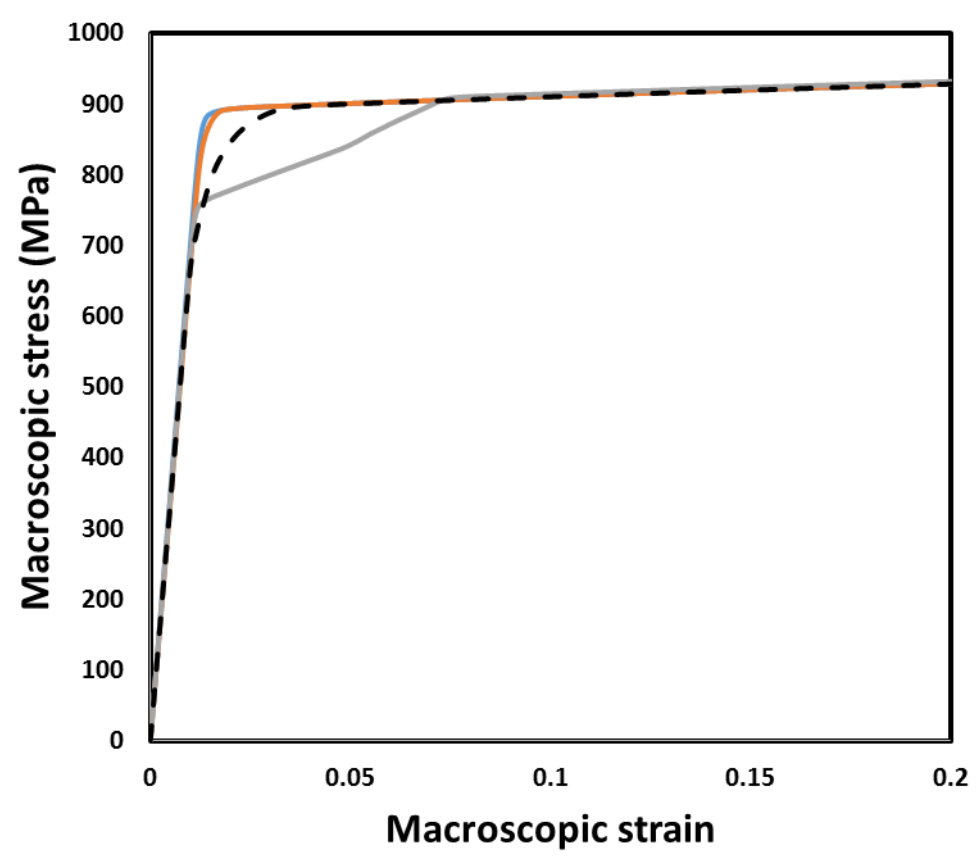

$$
\dot{\varepsilon}=2 * 10^{-4} s^{-1}
$$

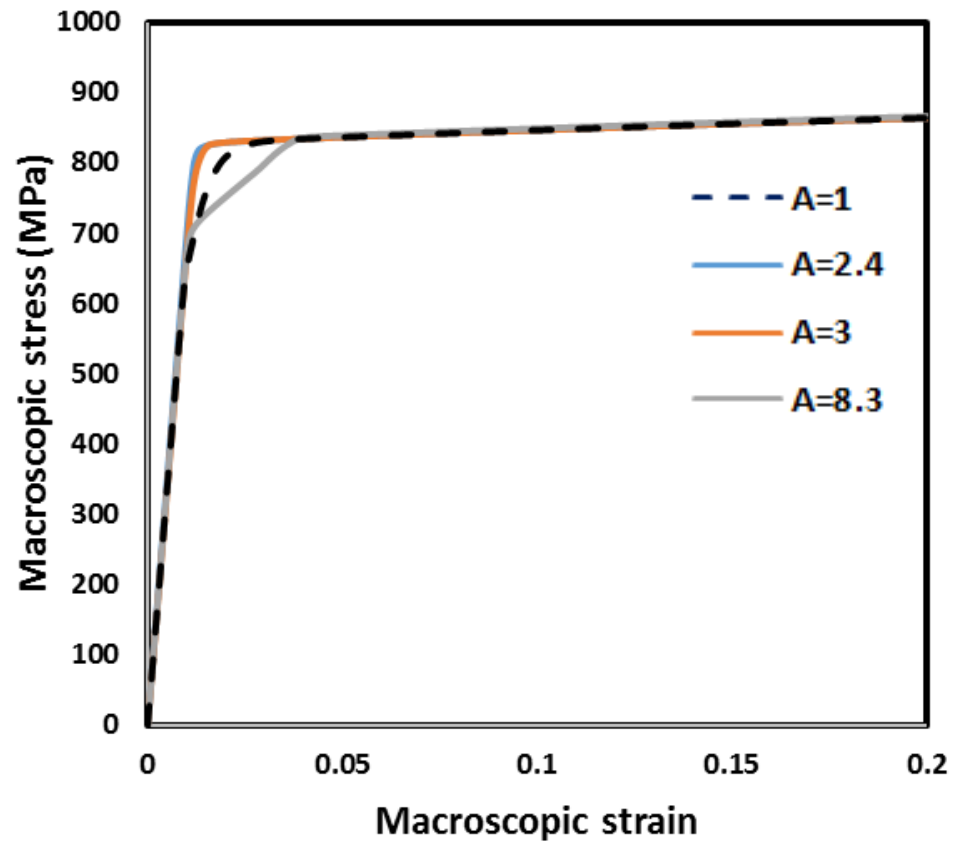

Figure 5- Tensile macroscopic stress $\left(\Sigma_{33}\right)$ vs macroscopic strain $\left(E_{33}\right)$ estimated by the Affine EVPSC model at two strain rates: $2 \times 10^{-3} s^{-1}$ and $2 \times 10^{-4} s^{-1}$ using four anisotropy coefficients (A) (equiaxed $\beta$ microstructure with $\beta$-forged texture). 


\subsection{Effect of elastic anisotropy on elastic-plastic incompatibility stresses for $<111>$ and $<100>$ grains}

\subsubsection{Equiaxed $\beta$ microstructure with random texture}

Incompatibility stresses are defined as the difference between the local mean stress $\left(\sigma_{33}\right)$ in a grain and the macroscopic one $\left(\Sigma_{33}\right)$. The predicted incompatibility stresses in $<111>$ and $<100>$ grains for random texture are plotted as a function of the macroscopic strain for $A=1,2.4,3$ and 8.3 (Fig. 6(a)). Several important results are reported:

(i) Incompatibility stresses increase linearly with the macroscopic strain in the elastic domain, then decrease progressively when plastic strain begins. Incompatibility stresses have a positive contribution in $<111>$ grains and a negative one in $<100>$ grains. This means that the highest local stresses are achieved in $<111>$ grains at the end of the elastic loading.

(ii) The development of incompatibility stresses during the elastic domain arises from the elastic anisotropy of the $\beta$-phase. As shown in Fig. 6(a) they depend on the value of $A$ : incompatibility stresses obtained for $A=8.3$ are the largest in magnitude for both $<100>$ and $<111>$ grains. For comparison, no incompatibility stresses are observed for $A=1$ in the elastic domain (see Fig. 5(a)). These latter develop later due to viscoplastic incompatibilities. Unlike the similar predicted macroscopic elasto-viscoplastic responses obtained for $A=2.4,3,8.3$ (as shown in Fig. 3), the predicted incompatibility stresses are different from each other during the elastic-viscoplastic transition even between $A=2.4$ and $A=3$.

(iii)Specifically for $<100>$ grains, the incompatibility stresses predicted for $A=8.3$ stabilize only at $\sim 4.5 \%$ macroscopic strain compared to $A=2.4$ and $A=3$ which stabilize earlier at $\sim 1.5 \%$ strain. Incompatibility stresses do not stabilize before $<100>$ grains become plastic as shown in Fig. $6(b)$. For $A=8.3$, the plastic strain starts only at $\sim 4.5 \%$ macroscopic strain while for $A=2.4$ and $A=3$, it starts earlier at $\sim 1.5 \%$. Regarding the $<111>$ grain, it is shown in Fig. $6(\mathrm{c})$ that its plastic strain starts at the same macroscopic strain of $\sim 1.3 \%$ for the three anisotropy coefficients $A=2.4,3,8.3$ but the one for $A=8.3$ increases a little faster. However, the plastic strain rate changes and becomes similar to that observed with $A=2.4$ and $A=3$ when plasticity occurs in the $<100>$ grain. Thus, the plastic strain of $<111>$ grain is only slightly more important for $A=8.3$ compared to $A=2.4$ and $A=3$. This explains why predicted incompatibility stresses generated in $<111>$ grains quickly converge to the same viscoplastic asymptotic state for all $A$ values compared to those predicted in $<100>$ grains.

\subsubsection{Equiaxed $\beta$ microstructure with $\beta$-forged texture}


The incompatibility stresses predicted for the $\beta$-forged texture are more important compared to those obtained for the random texture and increase with the anisotropy coefficient (Fig. 7(a)). The strain for which the incompatibility stresses in $<100>$ and $<111>$ grains reach the viscoplastic asymptotic state highly depends on $A$ and on the texture. For the low A values (2.4 and 3 ), the asymptote is reached at lower strains in presence of texture and inversely for the high $A$ value (8.3). As in the random texture case, the plastic strain evolution of $\langle 100\rangle$ and $\langle 111\rangle$ grains for $A=8.3$ is different from that of $A=2.4$ and 3 . The effect is however much more marked in the $\beta$ forged texture case. The difference of plastic strain with $A=2.4$ and $A=3$ is very significant:

1) the plastic strain of $\langle 100\rangle$ grain starts only at $\sim 7.4 \%$ macroscopic strain while it starts at $\sim 1.4 \%$ for $A=2.4$ and $A=3$ (Fig. $7(b)$ ).

2) the plastic strain of $\langle 111>$ grain evolves much faster before slowing down at the onset of plastic strain in the $\langle 100\rangle$ grain (Fig. $7(\mathrm{c})$ ). It is 1.4 times larger than those obtained with $A=2.4$ and $A=3$. 
(a)

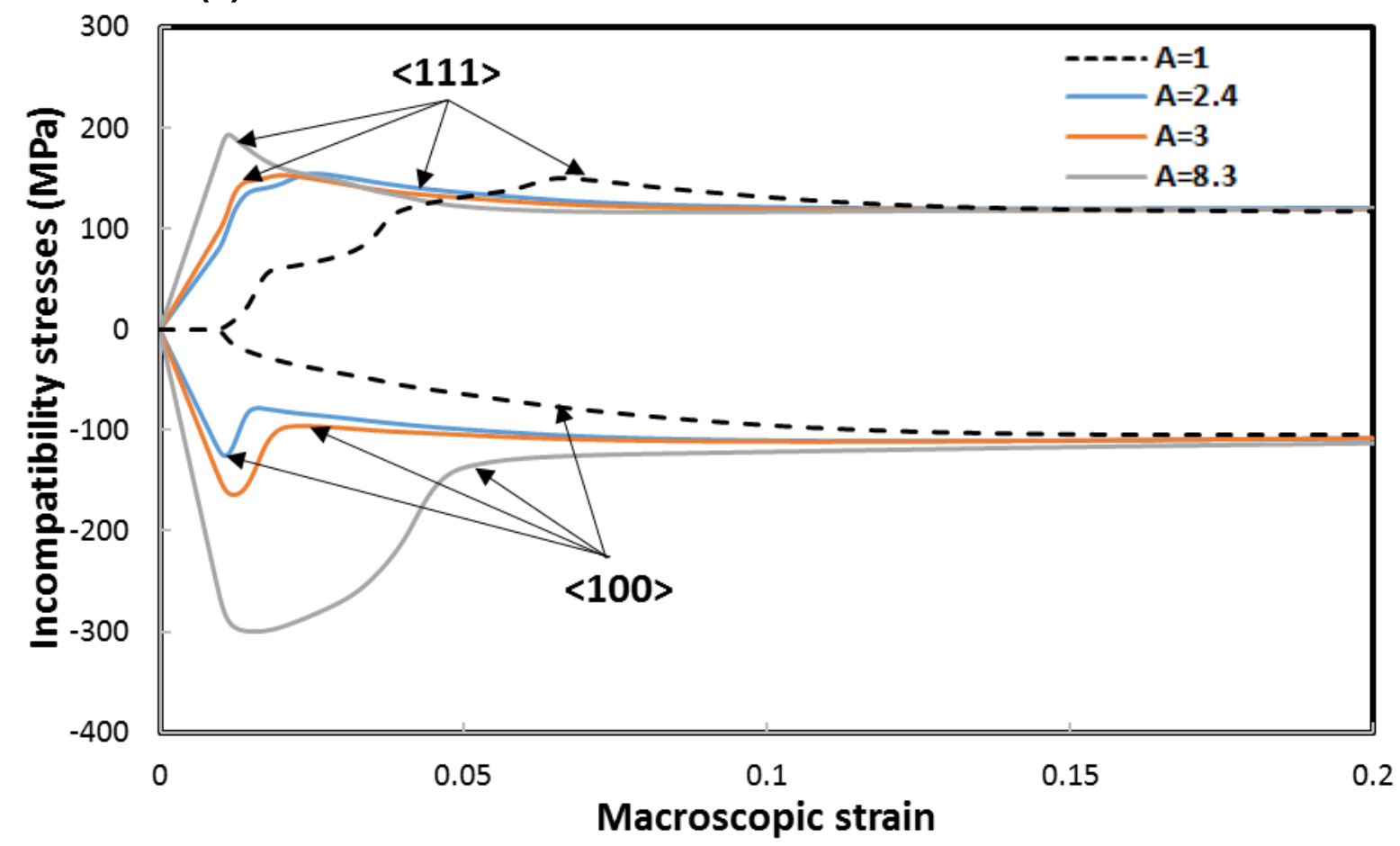

(b) Grain $\langle 100\rangle$

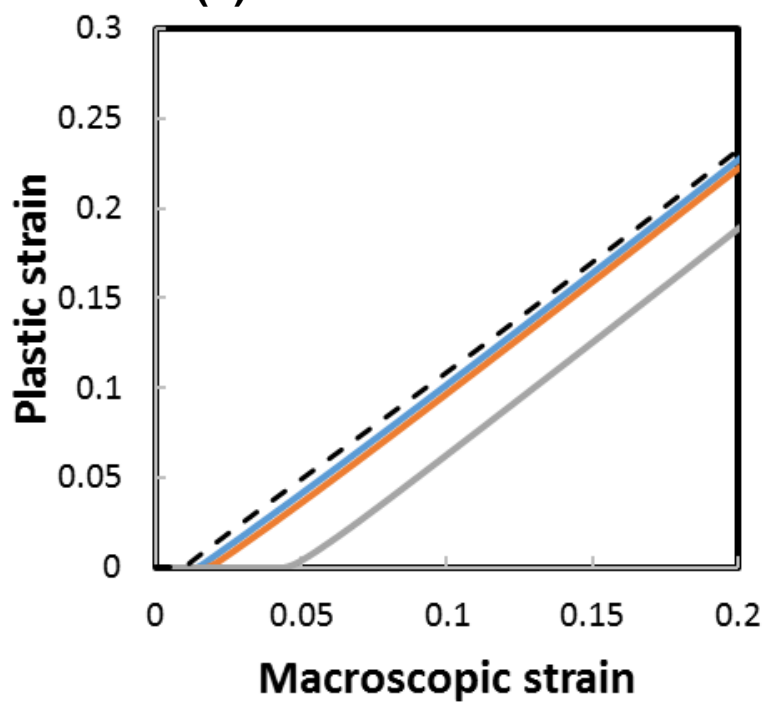

(c) Grain $\langle 111\rangle$

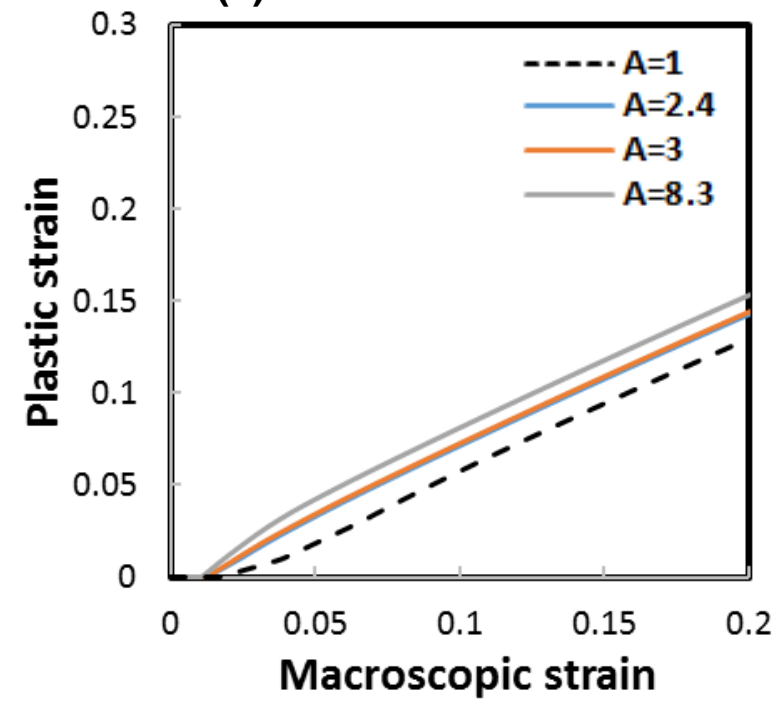

Figure 6- Incompatibility stresses $\left(\sigma_{33}-\Sigma_{33}\right)$ (a) and local plastic strain $\left(\varepsilon_{33}^{v p}\right)$ (b and c) as a function of the macroscopic strain $\left(E_{33}\right)$ of a $<100>$ grain and a $<111>$ grain. The numerical results are given for different anisotropy coefficients $A$ (equiaxed $\beta$ microstructure with random texture). 
(a)

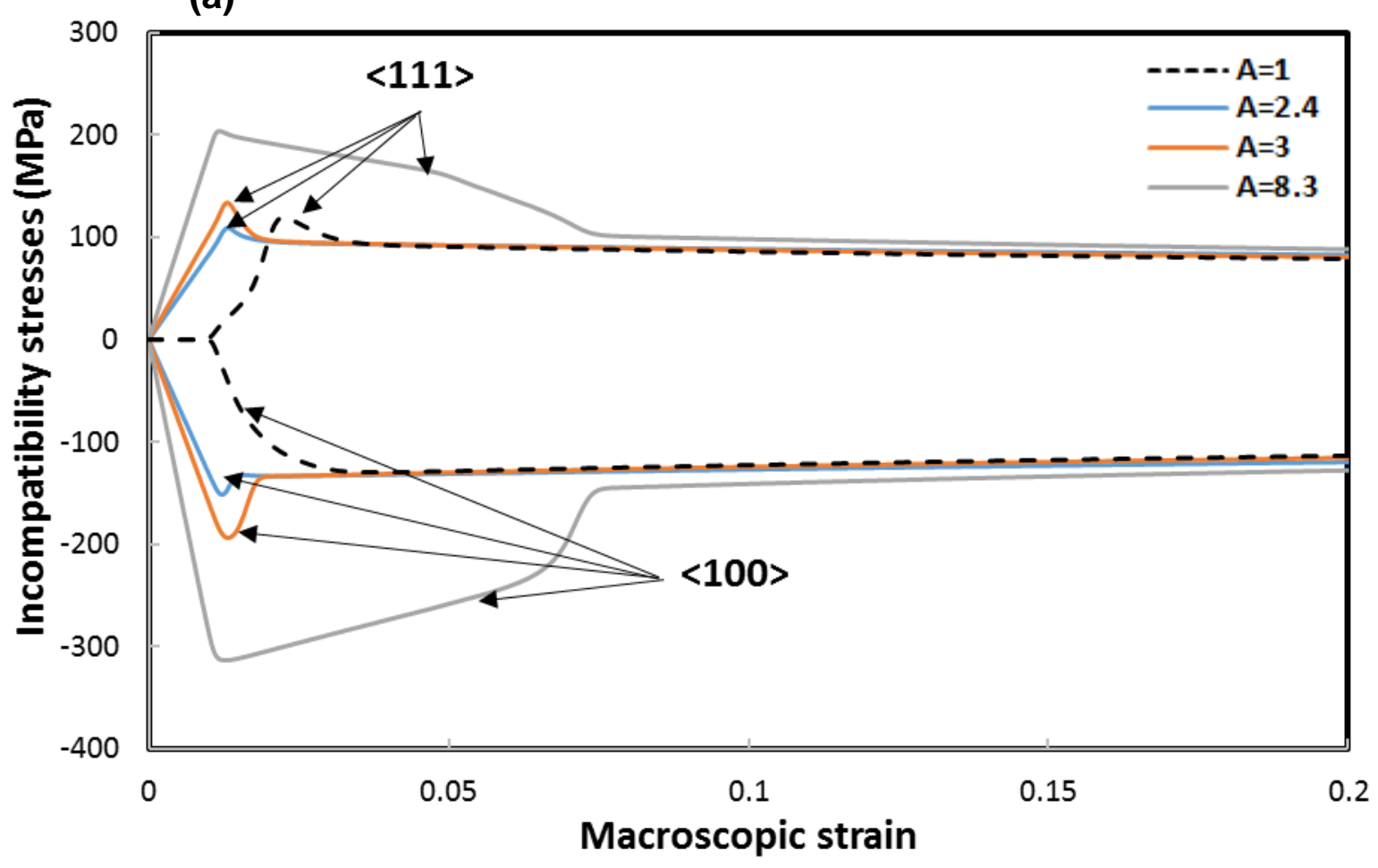

(b) Grain $<100\rangle$

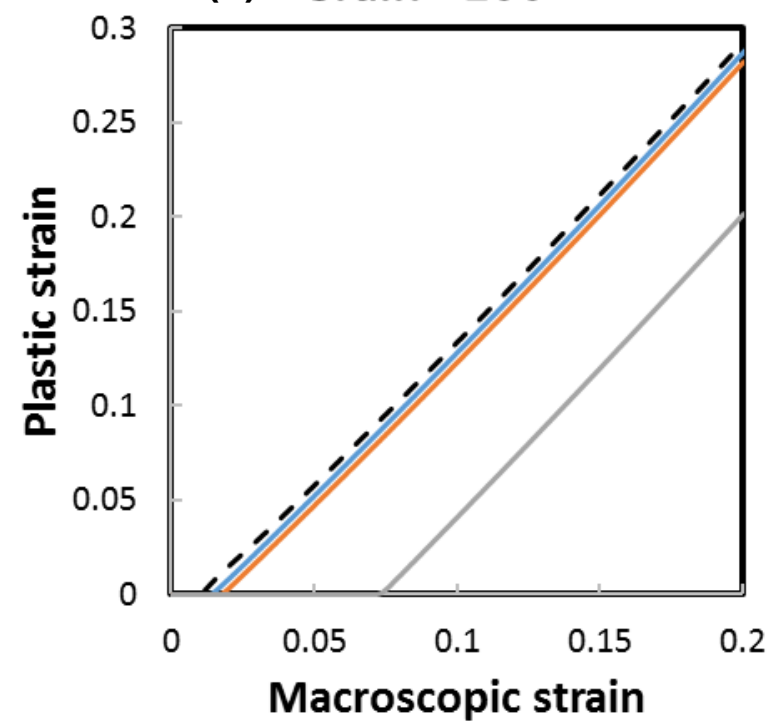

(c) Grain $\langle 111\rangle$

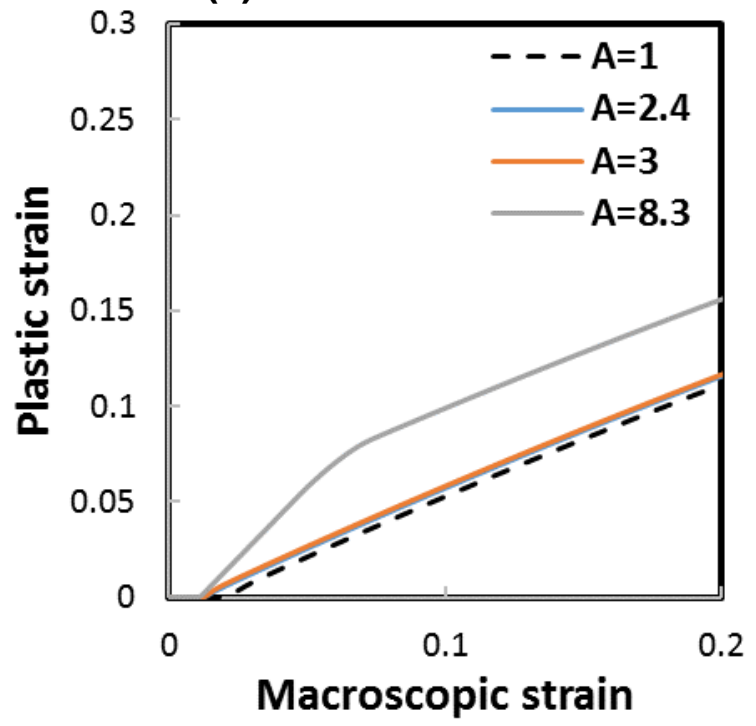

Figure 7- Incompatibility stresses $\left(\sigma_{33}-\Sigma_{33}\right)$ (a) and local plastic strain $\left(\varepsilon_{33}^{v p}\right)$ (b and c) as a function of the macroscopic strain $\left(E_{33}\right)$ of a $<100>$ grain and a $<111>$ grain. The numerical results are given for different anisotropy coefficients $A$ (equiaxed $\beta$ microstructure with $\beta$-forged texture). 


\subsection{Influence of elastic anisotropy on multiaxial plastic yielding}

In this part, we investigate through the plastic yield surfaces the influence of the elastic anisotropy on the overall plastic flow stress response for different multiaxial loading paths. Fig. 8 represents in the case of random texture the overall plastic yield loci in the $\left(\Sigma_{11}, \Sigma_{33}\right)$ plane at the onset of macroscopic plasticity, i.e. for a macroscopic equivalent viscoplastic strain of $\mathrm{E}^{\mathrm{vpe}}=$ $0.2 \%$. The yield surfaces are close to Von Mises ones. For $A=2.4,3$ and 8.3 , the yield surfaces are close from each other but undergo an expansion in size compared to the isotropic case $(A=1)$. Note that for isotropic elasticity, the yield surface is bounded by the grain having the strongest Schmid factor while for anisotropic elasticity, the yield surface is expanded because the grain with the strongest Schmid factor does not necessarily initiate the plasticity due to elastic incompatibilities (Cailletaud and Coudon, 2016; Sauzay, 2006).

We show for $A=3$ (Fig. 9(a)) and $A=8.3$ (Fig. 9(b)) that the obtained yield surfaces for the $\beta$ forged texture are strongly asymmetric with respect to the first diagonal axis $\left(\Sigma_{11}=\Sigma_{33}\right)$ and this effect is the most pronounced for $A=8.3$. Elastic and plastic incompatibilities can have a predominant influence on the initial yield loci. By examining Fig. 9(a) and $9(\mathrm{~b})$, it is observed that the yield stresses in the case of equibiaxial tensile or compressive loadings (i.e. along the first diagonal axis: $\Sigma_{11}=\Sigma_{33}$ ) obtained for a strongly anisotropic texture ( $\beta$-forged texture) are lower than those obtained for the random texture. In contrast, for "shear-type" loadings corresponding to $\Sigma_{11}=-\Sigma_{33}$, there is only a little difference between random and $\beta$-forged textures.

Hence, it is also interesting to notice that the yield surface is less sensitive to the $\beta$-forged texture when crystal elasticity is isotropic $(A=1)$. The initial yield surfaces obtained for the random texture and for the $\beta$-forged one are indeed close to each other (Fig. 9(c)). In this case, only the effect of plastic anisotropy on the yield loci at the onset of slip is demonstrated. Therefore, because of the low overall plastic strain $\left(\mathrm{E}^{\mathrm{vpe}}=0.2 \%\right.$ ), only a slight modification of the isotropic Von Mises-like yield surface is observed. For a $\beta$-forged texture, the yield stresses obtained for $A=1$ are more important than thoses for $A=3$ and $A=8.3$ in the case of equibiaxial tensile or compressive loadings. This was never observed when comparing the yield surfaces obtained for the random texture (Fig. 8). This effect is thus related to the combination of the anisotropic texture with heterogeneous elasticity. 


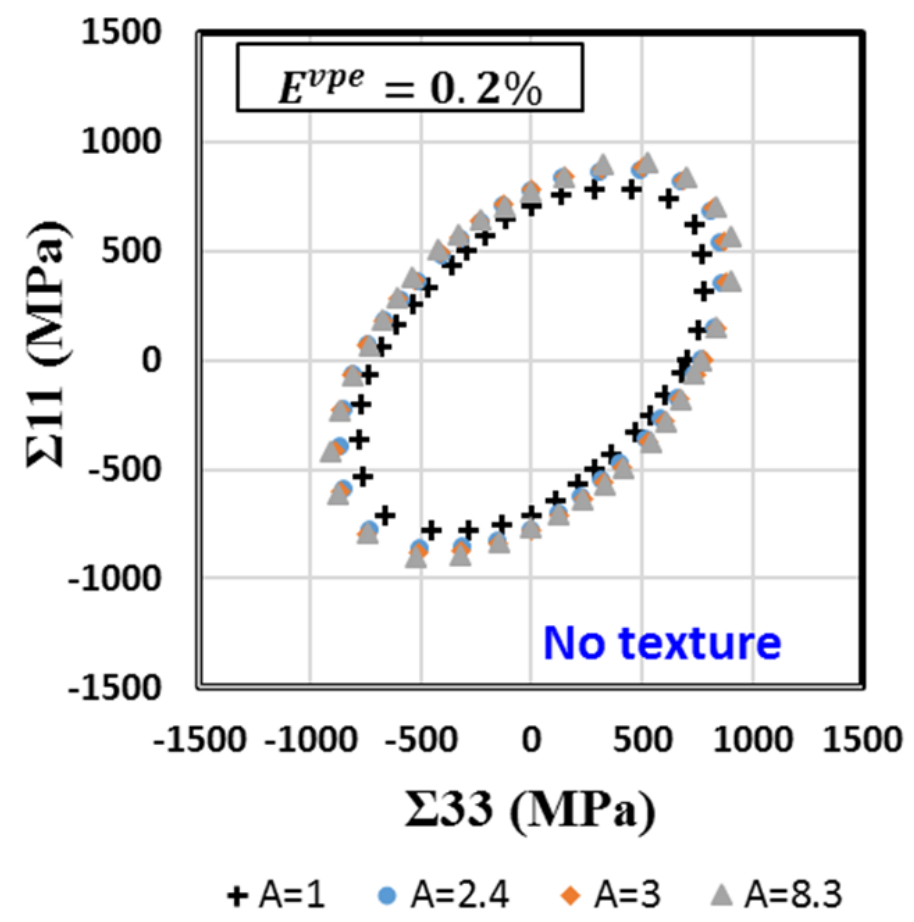

Figure 8- Simulated yield surfaces (yield loci) in the plane $\left(\Sigma_{11}, \Sigma_{33}\right)$ at macroscopic equivalent viscoplastic strain $E^{\text {vpe }}=0.2 \%$. The yield surfaces are plotted for different anisotropy coefficients (A) (equiaxed $\beta$ microstructure with random texture). 
(a)

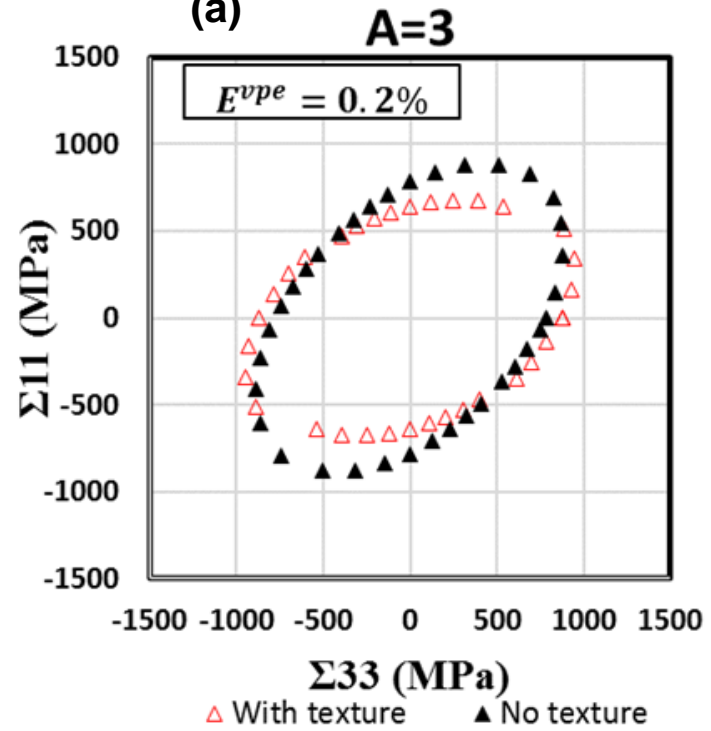

(b)

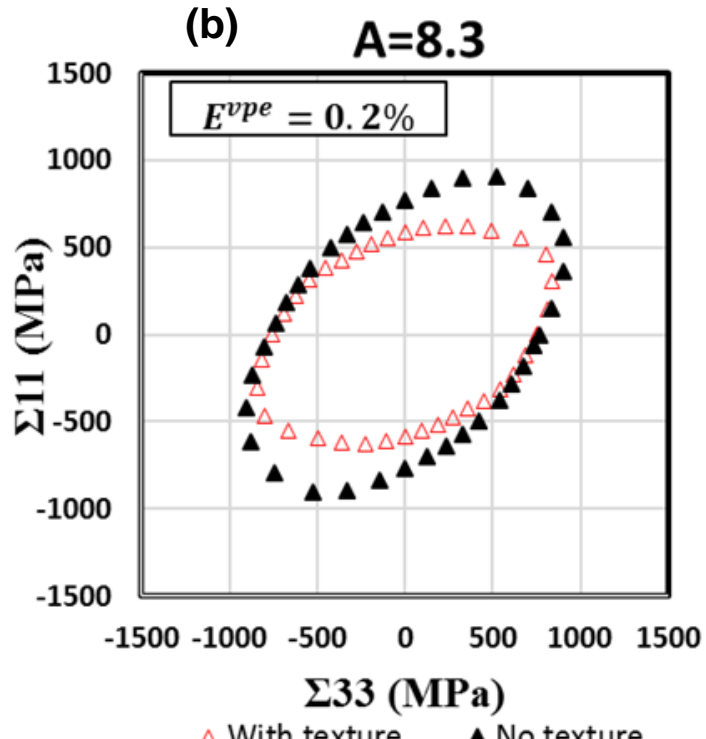

(c) $\quad A=1$

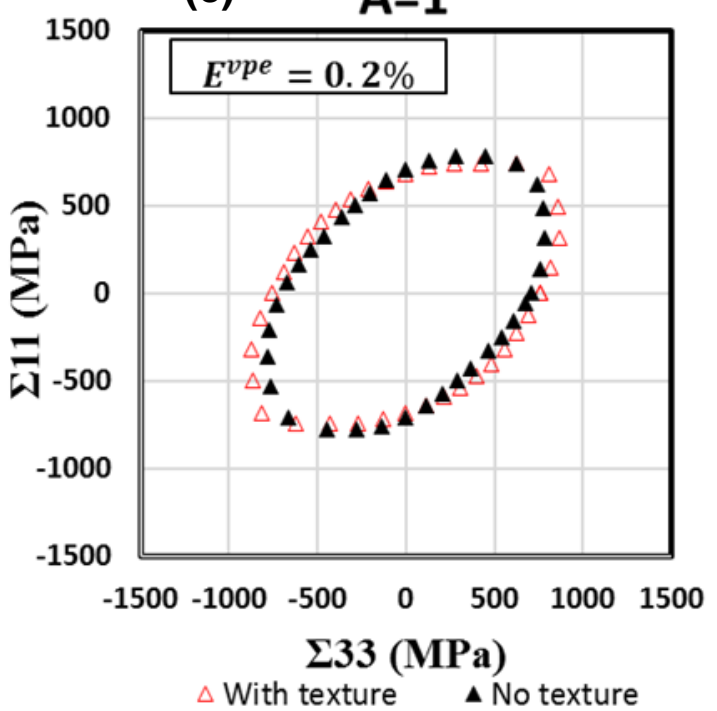

Figure 9- Initial yield surfaces in the plane $\left(\Sigma_{11}-\Sigma_{33}\right)$ at macroscopic equivalent viscoplastic strain $E^{v p e}=0.2 \%$. The yield loci are given for different anisotropy coefficients $(A)$ and for both random and $\beta$ forged textures.

\subsection{Effect of elastic anisotropy and crystallographic texture on cyclic responses: discussion on the Bauschinger effect}

The strong stress incompatibilities induced by the $\beta$-elastic anisotropy during unidirectional loading also affect the macroscopic $\beta$-Ti behavior under cyclic loading, as demonstrated in this section. Cyclic tension-compression loading simulations are performed at an applied strain rate of $2 \times$ $10^{-3} s^{-1}$, see Fig. 10. The cyclic test is strain-controlled with applied uniaxial strain $\mathrm{E}_{33}$ between 0.05 and +0.05 strain. One cycle is simulated with the affine-based EVPSC for the different SEC 
and for random texture (Fig. 10(a)) and $\beta$-forged texture (Fig. 10(b)). The objective is to study the effect of elastic and plastic anisotropies on the Bauschinger effect in $\beta$-Ti, which is of importance for metal forming operations of Ti alloys (Beal et al., 2006). For example, the Bauschinger effect was recently studied using reverse loading in-plane continuous tension-compression and compression-tension tests at room temperature on Ti-6Al-4V (Badr et al., 2016). The presence of the Bauschinger effect is at the origin of "kinematic hardening" during cyclic plasticity (Armstrong and Frederick, 1966; Cailletaud, 1992; Khan et Huang, 1995; Chaboche et al., 2012; Smith et al., 2018). The macroscopic "Bauschinger stress" characterizing the Bauschinger effect is a macroscopic signature of incompatibility stresses due to elastic and plastic inter-granular accommodation during tensile stage and their progressive rearrangement during subsequent compression stage. The Bauschinger effect originates from inter-granular strain/stress incompatibilities and from the c parameter used in the linear intra-granular kinematic hardening model. These incompatibility strains/stresses and the "Bauschinger stress" can be experimentally extracted and validated from lattice strain measurements using in situ neutron/synchrotron diffraction (Huang et al., 2012, 2015; Taupin et al., 2013; Hounkpati et al., 2016) and macroscopic tension-compression test (Taupin et al., 2013; Badr et al., 2016). This "Bauschinger stress" is usually defined as the difference between the forward tensile flow stress $\Sigma_{33}^{2}$ and the yield stress on reverse flow in compression $\Sigma_{33}^{1}$ as shown in Fig. 10(c), see e.g. Sowerby et al. (1979). Then, the equation defining the Bauschinger stress is $\Sigma_{33}^{2}-\Sigma_{33}^{1}$. Let us note that other definitions may exist in the literature (Lemoine and Aouafi, 2008).

From Fig. 10( $a$ and b), the Bauschinger stress is calculated for random and $\beta$-forged textures and described in Fig. 11(a and b), respectively. Two important results arise from theses simulations:

1) For a random texture, the Bauschinger stress monotonically increases with the $\beta$-elastic anisotropy coefficient A (Fig. 11(a)). This increase reaches 100MPa between the two extreme $A$ values at a loading level of $250 \mathrm{MPa}$ (238MPa for $A=1$ to $338 \mathrm{MPa}$ for $A=8.3$ ).

2) The $\beta$-forged texture reduces the Bauschinger stress for all considered $A(\neq 1)$ values (Fig. 11(b)). This results from a compensation effect between elastic and plastic sources of incompatibility stresses. Only in case of isotropic elasticity where no elastic stress incompatibilities arise, the $\beta$-forged texture increases the Bauschinger stress from $238 \mathrm{MPa}$ (random texture) to $435 \mathrm{MPa}$ (see $A=1$, Fig. 11(a,b)). This value is about twice higher than for the anisotropic elastic cases for which $\Sigma_{33}^{2}-\Sigma_{33}^{1}=170 \mathrm{MPa}, 226 \mathrm{MPa}$ and $217 \mathrm{MPa}$ for $A=2.4,3$ and 8.3 , respectively. For $A=1$, the strong crystallographic texture is at the origin of the increase of the Bauschinger stress. 

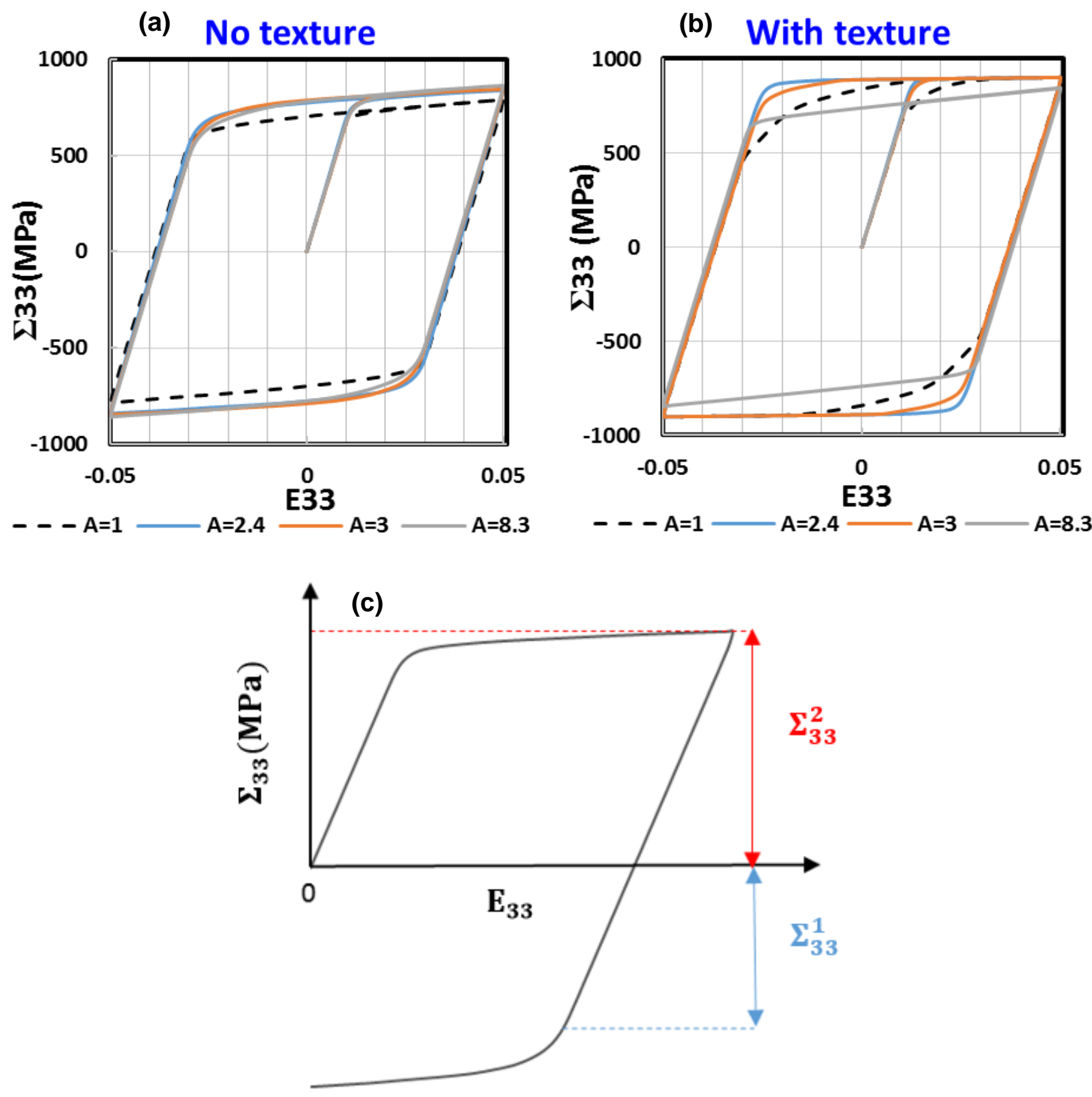

Figure 10- Influence of $\beta$ elastic anisotropy on stress-strain curves under strain controlled cyclic loadings with strain amplitude of $-/+5 \%$. Results are given for different anisotropy coefficients (A). (a) Random texture, (b) $\beta$-forged texture. (c) Schematic picture defining the overall Bauschinger stress as $\Sigma_{33}^{2}-$ $\Sigma_{33}^{1}$ from the macroscopic stress-strain curve. 
(a)

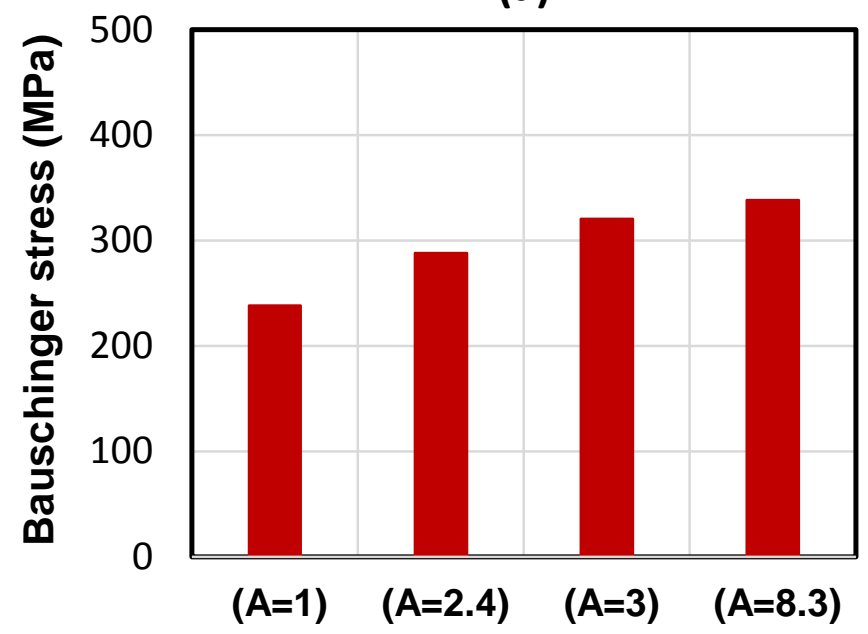

(b)

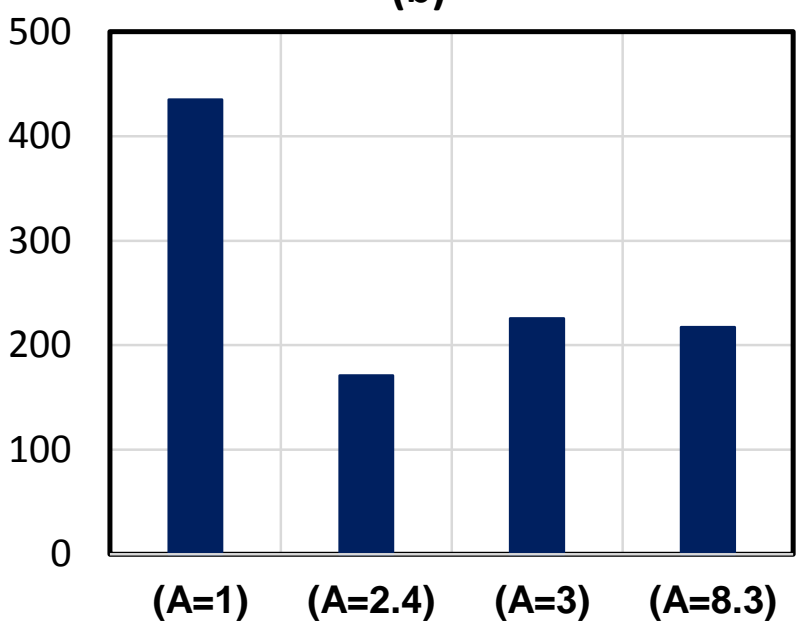

Figure 11- Predicted Bauschinger stress $\left(\Sigma_{33}^{2}-\Sigma_{33}^{1}\right)$ for $A=1,2.4,3$ and 8.3 for (a) random and (b) $\beta$-forged textures after one cycle of $+/-0.05$ strain controlled tensile/compression loading

5.5. Effect of the $\beta$-phase grain morphology and SEC on elastic-plastic incompatibilities in $<100>$ and $<111>$ grains

The prior $\beta$-grain shape in titanium alloys exhibits often an ellipsoidal shape induced by the hot forging process as illustrated in Fig. 12. An EBSD (Electron Back Scattered Diffraction) map obtained from Scanning Electron Microscope (SEM) is reported showing the elongated shape of $\beta$ grains in the axial direction (AD) of the forged product (Chini et al., 2016b). From this figure, it is shown that ellipsoidal grains with an average aspect ratio of 3 are realistic for prior $\beta$ grains. Therefore, oblate and prolate spheroids with an aspect ratio of 3 are studied with the present crystal plasticity based EVPSC scheme. 

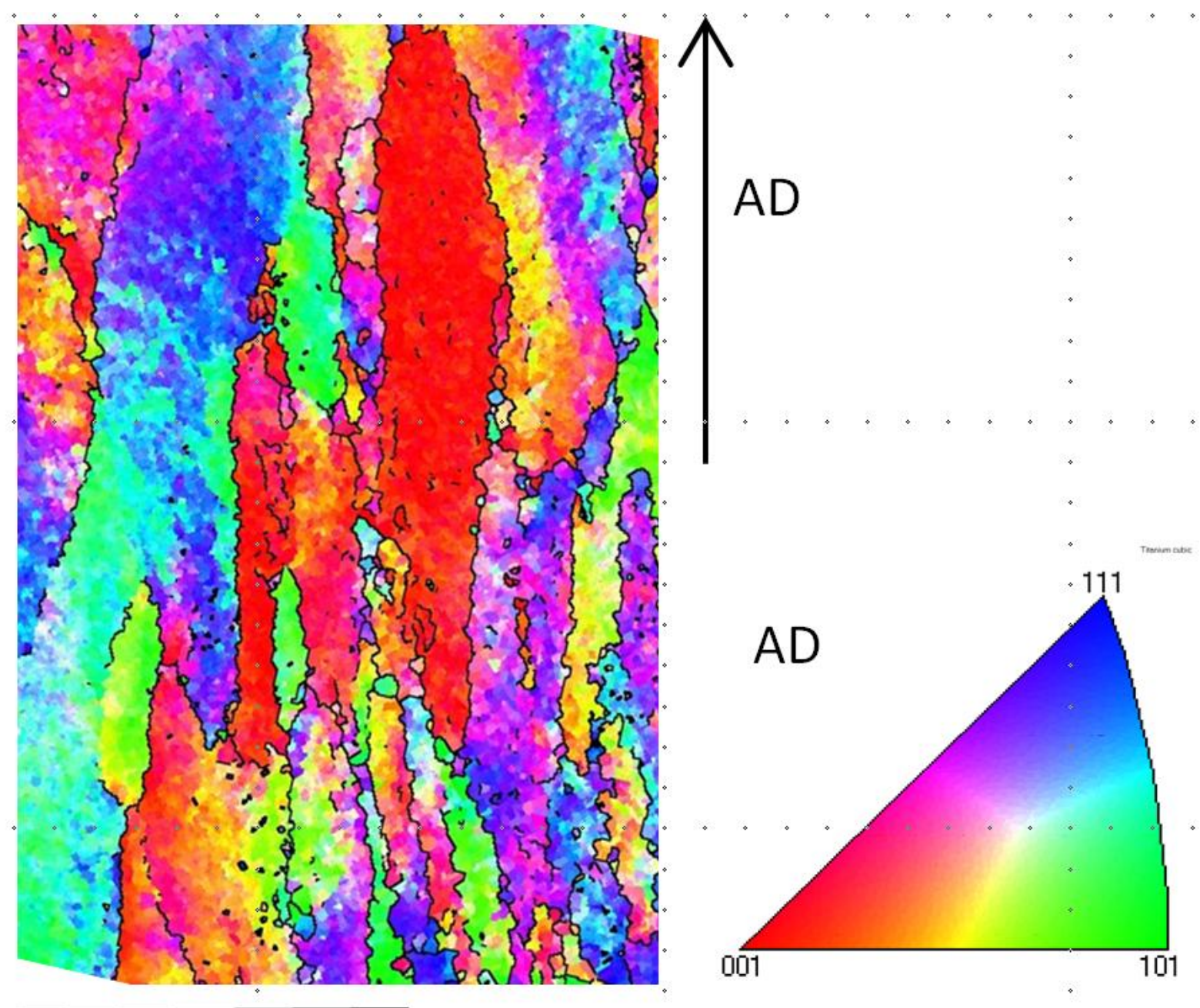

$1 \mathrm{~mm}$

Figure 12- EBSD map showing prior $\beta$ grains with an ellipsoidal shape in a forged titanium alloy (data from Chini et al. 2016b). The orientation color code is given by the attached Inverse Pole Figure//Axial Direction (AD) of the forged product.

The simulations show that the $\beta$-grain morphology has a negligible effect on the overall elastoviscoplastic response for all coefficients $A$ (curves are not shown here). However, again it strongly modifies the local grain incompatibility stresses and thus may affect the final mechanical behavior of $\beta-\mathrm{Ti}$ alloys. This is illustrated in Fig. 13 that gives the incompatibility stresses predicted for $<111>$ and $<100>$ grains taken from our set of randomly oriented grains subjected to uniaxial tension. Three grain shapes are considered in the EVPSC model: (i) spheres ( $b / a=c / a=1)$, (ii) oblate spheroids $(\mathrm{a} / \mathrm{c}=\mathrm{b} / \mathrm{c}=3)$ or (iii) prolate spheroids $(\mathrm{c} / \mathrm{a}=\mathrm{c} / \mathrm{b}=3)(\mathrm{a}, \mathrm{b}$ and $\mathrm{c}$ are the half-axes of the ellipsoidal inclusions and the major axes are either oriented parallel ("LONG") or perpendicular ("TRANS") to the loading direction).

The highest magnitude of incompatibility stresses between $<111>$ and $<100>$ grains are reported for 'LONG'-oriented prolate spheroidal grains and the lowest for 'TRANS'-oriented oblate and 
prolate spheroidal grains, whatever the elastic anisotropy (spherical $\beta$-grains constitute an intermediate case). For example, the incompatibility stresses obtained with "LONG"-oriented prolate spheroids are about two to three times those obtained with "TRANS"-oriented oblate spheroids for $A=2.4$ and $A=3$ and up to four times for $A=8.3$ at the end of the elastic regime.

Again, the higher the elastic anisotropy coefficient, the higher the magnitude of incompatibility stresses between $<111>$ and $<100>$ grains. 

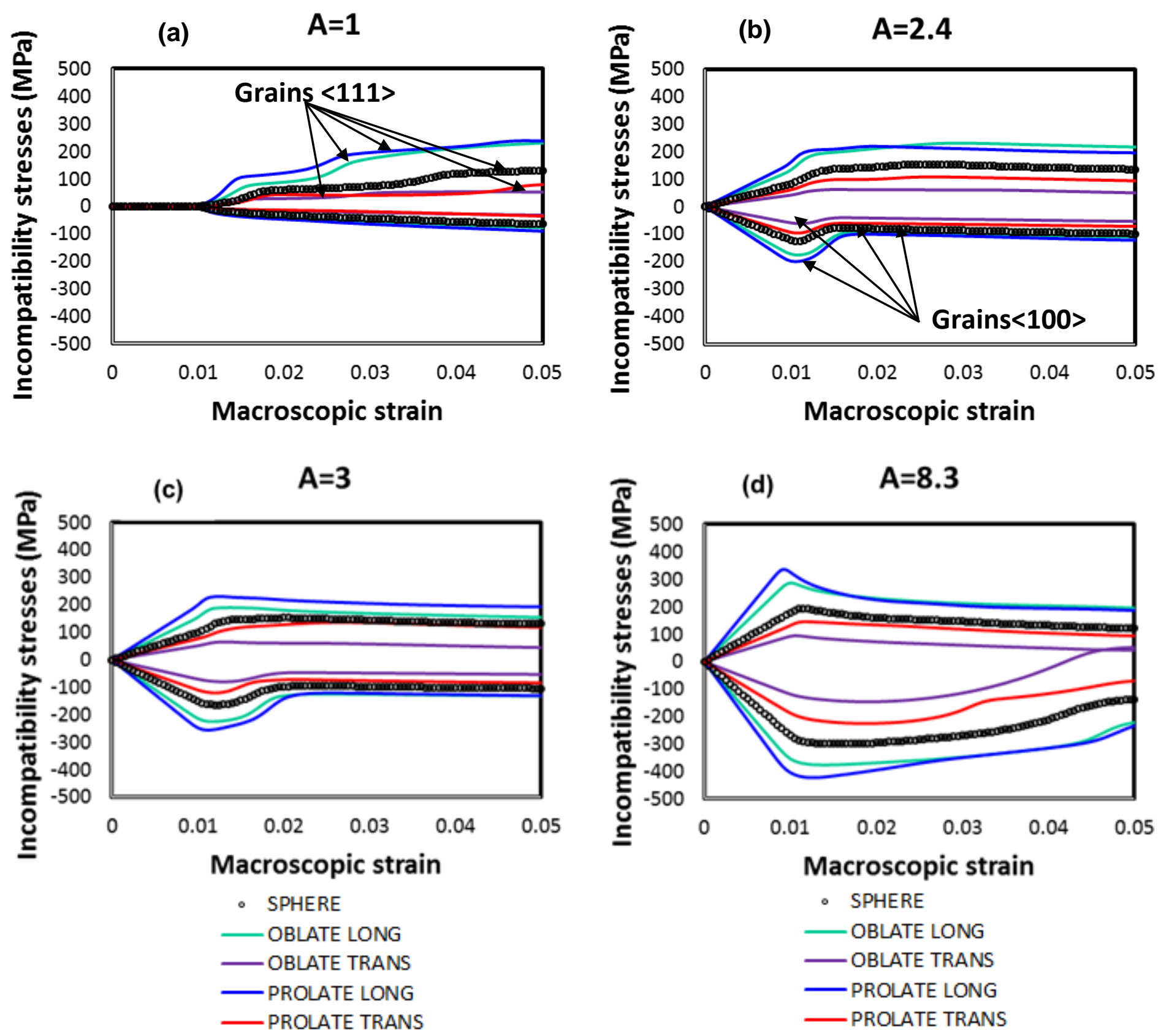

Figure 13- Incompatibility stresses in $<100>$ and $<111>$ grains computed for different grain shapes and anisotropy coefficients (A). The simulations are performed with our set of randomly oriented grains subjected to uniaxial tension. 


\section{Conclusions and perspectives}

The $\beta$-phase of titanium is known to exhibit an anisotropic elastic behavior. However, the way how this elastic anisotropy impacts the mechanical behavior in fully $\beta$-microstructures or in more complex $\alpha / \beta$ microstructures is still an open question. This topic is even complicated by the uncertainties on the $\beta$ phase SEC: $\mathrm{C}_{11}, \mathrm{C}_{12}$ and $\mathrm{C}_{44}$ with published values corresponding to a large set of anisotropy coefficients.

In this contribution, a recent affine elasto-viscoplastic self-consistent (EVPSC) model (Mareau and Berbenni, 2015) was applied to theoretically investigate the elastic-viscoplastic behavior of $\beta$ phase titanium (such as Ti-17 or Ti-5553) and to capture elastic/viscoplastic incompatibilities. The model is based on the affine linearization of the viscoplastic flow rule. This allows a good description of the overall and the mean local responses of elastically anisotropic $\beta$-titanium polycrystals with highly nonlinear viscoplastic flow rule at the scale of slip systems. The EVPSC model was especially applied to capture coupled effects of $\beta$ elastic anisotropy, $\beta$ - grain shape and texture on overall stress response and inter-granular incompatibility stresses. Three different sets of the SEC extracted from the literature and corresponding to Zener coefficients $A=2.4,3$ and 8.3 were considered in the simulations as they all give the experimental Young's modulus of the studied alloy (Ti-17). The following conclusions can be drawn from this work:

1. The experimental macroscopic tensile elasto-viscoplastic transition of a random oriented and equiaxed $\beta$ Ti-17 is well predicted by the model for two strain rates. This demonstrates the ability of the model to capture the elastic-viscoplastic transition of this strain rate sensitive alloy and its reliability for further predictive simulations.

2. Whatever the considered SEC, the macroscopic predicted responses are similar for randomly oriented $\beta$ phase grains, equiaxed or ellipsoidal in shape. This clearly indicates that considering only the macroscopic behavior is not discriminant to evaluate the elastic anisotropy of the $\beta$ phase. On contrary, the model indicates that in presence of a sharp $\beta$-forged texture, differences are observed.

3. As a major result, the three considered sets of SEC imply strong variations in incompatibility stresses between differently oriented $\beta$-grains with lowest and highest values obtained respectively for $<100>$ and $<111>$ grains (fiber orientation parallel to the loading direction). This magnitude increases with the Zener anisotropy coefficient, with the $\beta$-forged texture and with the ellipsoidal (non spherical) grain shape where major axis is oriented parallel to the loading direction. 
4. These incompatibility stresses are also of major importance to understand the low-cycle fatigue behavior of near $\beta$-Ti alloys. The effect of anisotropy coefficient $A$ was seen to be important on the overall Baushinger stress reported from one cycle simulations. This Baushinger stress is a macroscopic signature of incompatibility stress development and inter-granular accommodation for the studied $\beta$-Ti alloy.

5. The plastic yield loci described for the three sets of SEC and coupled with a $\beta$-forged texture are remarkably modified for specific multiaxial loading directions (e.g., equibiaxial tension) compared to the ones obtained for the random texture. This was not observed when comparing the two yield surfaces in the context of isotropic elasticity only.

Finally, we draw attention to the major role of elastic anisotropy of $\beta$-phase in building up of incompatibility stresses that finally control the material's behavior during uniaxial, reverse and cyclic loadings. Consequently, determining or refining the real SEC of the $\beta$-phase are fundamental to predict the local incompatibility stresses and in use effective properties of near $\beta-\mathrm{Ti}$ alloys with simple $\beta$ microstructures or more complex $\alpha / \beta$ microstructures in a future work. The simulations demonstrate also the coupled effects of texture and grain shape with the $\beta$ elastic anisotropy. They may serve to design optimized $\beta$-grain shape (spheres, oblate ellipsoids, prolate ellipsoids) and crystallographic orientation distributions for reducing stress concentrations for given loading directions. 


\section{Appendix A}

\section{A.1. Cases of pure elasticity and pure viscoplasticity with affine formulation}

The properties obtained from the averaging equations of homogenization theory $(\langle\dot{\boldsymbol{\varepsilon}}\rangle=\dot{\mathbf{E}},\langle\dot{\boldsymbol{\sigma}}\rangle=\dot{\boldsymbol{\Sigma}}$ and $\langle\boldsymbol{\sigma}\rangle=\boldsymbol{\Sigma})$ are given in the following but let us recall at first the expressions of the quantities $\dot{\boldsymbol{\varepsilon}}, \dot{\boldsymbol{\Sigma}}, \boldsymbol{\Sigma}, \dot{\boldsymbol{\sigma}}$ and $\boldsymbol{\sigma}$ :

$\dot{\boldsymbol{\varepsilon}}=\boldsymbol{A}^{\boldsymbol{C}}: \dot{\boldsymbol{E}} \quad$ for pure elasticity

$\dot{\varepsilon}=A^{B_{t}}: \dot{\boldsymbol{E}}+\boldsymbol{A}^{\boldsymbol{B}_{t}}: \boldsymbol{\Gamma}^{\boldsymbol{B}_{t}} *\left(\boldsymbol{b}_{\boldsymbol{t}}: \dot{\boldsymbol{\eta}}\right) \quad$ for pure viscoplasticity with affine approximation

The macroscopic constitutive laws display the same structure as the local constitutive ones, so:

$\dot{\boldsymbol{\Sigma}}=\boldsymbol{C}^{e}: \dot{\boldsymbol{E}} \quad$ for pure elasticity

$\Sigma=B_{t}^{e}:\left(\dot{E}-\dot{N}^{e}\right) \quad$ for pure viscoplasticity with affine approximation

The stress (stress rate) concentration rules are deduced from relations (12) and (13):

$\dot{\sigma}=\boldsymbol{c}: \boldsymbol{A}^{C}: \boldsymbol{S}^{\boldsymbol{e}}: \dot{\boldsymbol{\Sigma}} \quad$ for pure elasticity

$\sigma=b_{t}: A^{B_{t}}: M_{t}^{e}: \Sigma+b_{t}: A^{B_{t}}: \dot{N}^{e}+b_{t}: A^{B_{t}}: \Gamma^{B_{t}} *\left(b_{t}: \dot{\eta}\right)-b_{t}: \dot{\eta} \quad \begin{array}{r}\text { for pure viscoplasticity } \\ \text { with affine approximation }\end{array}$

The properties deduced from the averaging conditions are:

$C^{e}=\left\langle c: A^{C}\right\rangle$

$B_{t}^{e}=\left\langle b_{t}: A^{B_{t}}\right\rangle$

$\left\langle A^{C}\right\rangle=I$

$\left\langle A^{B_{t}}\right\rangle=I$

$\left\langle A^{B_{t}}: \Gamma^{B_{t}} *\left(b_{t}: \dot{\eta}\right)\right\rangle=\mathbf{0}$

$\left\langle b_{t}: \dot{\eta}\right\rangle-B_{t}^{e}: \dot{N}^{e}=\left\langle b_{t}: A^{B_{t}}: \Gamma^{B_{t}} *\left(b_{t}: \dot{\eta}\right)\right\rangle$

In addition, from averaging conditions over the RVE applied to equations (19-22), we obtain:

$\left\langle\dot{\varepsilon}^{*}\right\rangle=\dot{\boldsymbol{E}}^{*}=\mathbf{0}$ 
$\left\langle\boldsymbol{s}: \dot{\boldsymbol{\sigma}}^{\prime}\right\rangle=\mathbf{0}$

$\left\langle\boldsymbol{m}_{t}: \sigma^{\prime \prime}\right\rangle=\mathbf{0}$

Subsequently, the following averaging relations are verified from averaging equations of the homogenization scheme $(\langle\dot{\boldsymbol{\varepsilon}}\rangle=\dot{\mathbf{E}},\langle\dot{\boldsymbol{\sigma}}\rangle=\dot{\boldsymbol{\Sigma}}$ and $\langle\boldsymbol{\sigma}\rangle=\boldsymbol{\Sigma})$ and from equations (A7-A12):

$\boldsymbol{S}^{e}:\left\langle\dot{\boldsymbol{\sigma}}^{\prime}\right\rangle=\boldsymbol{S}^{e}: \dot{\boldsymbol{\Sigma}}-\left\langle\dot{\boldsymbol{\varepsilon}}^{e}\right\rangle=\left\langle\dot{\boldsymbol{\varepsilon}}^{v p}\right\rangle-\dot{\boldsymbol{E}}^{v p, e}$

$M_{t}^{e}:\left\langle\sigma^{\prime \prime}\right\rangle=M_{t}^{e}: \Sigma+\dot{N}^{e}-\left\langle\dot{\varepsilon}^{v p}\right\rangle$

\section{A.2. Application of the self-consistent condition on the TF solution}

Using the decomposition of the modified Green operators into local and non-local parts and introducing unknown uniform tensors $\left(\mathbf{Y}_{\mathbf{1}}\right.$ to $\left.\mathbf{Y}_{\mathbf{4}}\right)$ in the integral equations (20-22) leads to:

$$
\begin{aligned}
& \dot{\boldsymbol{e}}^{\prime}=\left\langle\dot{\varepsilon}^{e}\right\rangle-\Gamma_{l}^{C}:\left(\delta c: \dot{e}^{\prime}\right)-\Gamma_{n l}^{C} *\left(\delta c: \dot{e}^{\prime}\right) \\
& \dot{\mathbf{e}}^{\prime \prime}=\left\langle\dot{\boldsymbol{\varepsilon}}^{\mathrm{vp}}\right\rangle-\Gamma_{1}^{\mathbf{B}_{\mathrm{t}}}:\left(\delta \mathbf{b}_{\mathrm{t}}: \dot{\mathrm{e}}^{\prime \prime}-\mathbf{b}_{\mathrm{t}}: \dot{\boldsymbol{\eta}}+\mathbf{B}_{\mathrm{t}}: \mathbf{Y}_{\mathbf{1}}\right)-\Gamma_{\mathrm{nl}}^{\mathrm{B}_{\mathrm{t}}} *\left(\delta \mathbf{b}_{\mathrm{t}}: \dot{\mathbf{e}}^{\prime \prime}-\mathbf{b}_{\mathrm{t}}: \dot{\boldsymbol{\eta}}+\mathbf{B}_{\mathrm{t}}: \mathbf{Y}_{\mathbf{1}}\right) \\
& \dot{\boldsymbol{\varepsilon}}^{*}=\dot{\mathbf{E}}^{*}-\Gamma_{1}^{\mathrm{C}}:\left(\delta \mathrm{c}: \mathbf{s}: \dot{\boldsymbol{\sigma}}^{\prime}-\mathbf{c}: \mathbf{A}^{\mathrm{C}}: \mathbf{Y}_{2}\right)-\Gamma_{\mathrm{nl}}^{\mathrm{C}} *\left(\delta \mathrm{c}: \mathbf{s}: \dot{\boldsymbol{\sigma}}^{\prime}-\mathrm{c}: \mathbf{A}^{\mathrm{C}}: \mathbf{Y}_{2}\right) \\
& -\Gamma_{1}^{B_{t}}:\left(\delta b_{t}: m_{t}: \sigma^{\prime \prime}-b_{t}: A^{B_{t}}: Y_{3}\right)-\Gamma_{n l}^{B_{t}} *\left(\delta b_{t}: m_{t}: \sigma^{\prime \prime}-b_{t}: A^{B_{t}}: Y_{3}\right) \\
& +\left(\Gamma_{1}^{C}: C-\Gamma_{1}^{B_{t}}: B_{t}\right):\left(m_{t}: \sigma^{\prime \prime}-A^{B_{t}}: Y_{4}\right)+\left(\Gamma_{n l}^{C}: C-\Gamma_{n l}^{B_{t}}: B_{t}\right) \\
& *\left(\mathbf{m}_{\mathbf{t}}: \boldsymbol{\sigma}^{\prime \prime}-\mathbf{A}^{\mathbf{B}_{\mathrm{t}}}: \mathbf{Y}_{\mathbf{4}}\right)
\end{aligned}
$$

Imposing the self-consistency conditions by neglecting the non-local terms and using equations (A7-A17) leads to:

$\left\langle\delta c: \dot{e}^{\prime}\right\rangle=0 \Rightarrow C=C^{e}$

$\left\langle\delta b_{t}: \dot{e}^{\prime \prime}-b_{t}: \dot{\eta}+B_{t}: Y_{1}\right\rangle=0 \Rightarrow B_{t}: Y_{1}=B_{t}^{e}: \dot{N}^{e}$

$\left\langle\delta c: s: \dot{\sigma}^{\prime}-c: A^{C}: Y_{2}\right\rangle=0 \Rightarrow Y_{2}=S^{e}:\left\langle\dot{\sigma}^{\prime}\right\rangle$

$\left\langle\delta b_{t}: m_{t}: \sigma^{\prime \prime}-b_{t}: A^{B_{t}}: Y_{3}\right\rangle=0 \Rightarrow Y_{3}=M_{t}^{e}:\left\langle\sigma^{\prime \prime}\right\rangle$

$\left\langle m_{t}: \sigma^{\prime \prime}-A^{B_{t}}: Y_{4}\right\rangle=0 \Rightarrow Y_{4}=0$ 


\section{A.3. Expressions of the effective viscoplastic strain rate $\dot{\mathbf{E}}^{\mathrm{vp}, \mathrm{e}}$ and back- extrapolated strain rate $\dot{\mathrm{N}}^{\mathrm{e}}$}

The effective viscoplastic strain rate $\dot{\mathbf{E}}^{\text {vp,e }}$ which is chosen to impose the condition $\langle\dot{\boldsymbol{\sigma}}\rangle=\dot{\boldsymbol{\Sigma}}$ is obtained as follows:

$$
\begin{aligned}
\dot{E}^{v p, e}=Z:\langle c: & \left.\dot{\varepsilon}^{v p}\right\rangle-Z:\left\langle c: A^{C}: \Gamma_{l}^{B_{t}}:\left(b_{t}: \dot{\eta}-B_{t}^{e}: \dot{N}^{e}\right)\right\rangle \\
& -Z:\left\langle c: A^{C}: \Gamma_{l}^{C}:\left(\delta c: \dot{\varepsilon}^{v p}+c: A^{C}:\left\langle\dot{\varepsilon}^{v p}\right\rangle\right)\right\rangle \\
& +Z:\left\langle c: A^{C}: \Gamma_{l}^{B_{t}}:\left(\delta b_{t}: \dot{\varepsilon}^{v p}-b_{t}: A^{B_{t}}:\left(M_{t}^{e}: \Sigma+\dot{N}^{e}-\left\langle\dot{\varepsilon}^{v p}\right\rangle\right)\right)\right\rangle \\
& -Z:\left\langle c: A^{C}:\left(\Gamma_{l}^{C}: C^{e}-\Gamma_{l}^{B_{t}}: B_{t}^{e}\right):\left(\dot{\varepsilon}^{v p}-A^{B_{t}}:\left\langle\dot{\varepsilon}^{v p}\right\rangle\right)\right\rangle \\
& +Z:\left\langle c: A^{C}:\left(\Gamma_{l}^{C}: C^{e}-\Gamma_{l}^{B_{t}}: B_{t}^{e}\right):\left(A^{B_{t}}: \Gamma_{l}^{B_{t}}:\left(b_{t}: \dot{\eta}-B_{t}^{e}: \dot{N}^{e}\right)\right)\right\rangle
\end{aligned}
$$

with:

$Z=\left(I-S^{e}:\left\langle c: A^{C}: \Gamma_{l}^{C}: c: A^{C}\right\rangle\right)^{-1}: S^{e}$

From Eq. (A12) and Eq. (A22), the effective back-extrapolated strain rate $\dot{\mathbf{N}}^{\mathrm{e}}$ is given by:

$\mathbf{B}_{\mathbf{t}}^{\mathbf{e}}: \dot{\mathbf{N}}^{\mathbf{e}}=\left\langle\mathbf{b}_{\mathbf{t}}: \dot{\boldsymbol{\eta}}-\mathbf{b}_{\mathbf{t}}: \mathbf{A}^{\mathbf{B}_{\mathrm{t}}}: \mathbf{\Gamma}_{\mathbf{l}}^{\mathbf{B}_{\mathrm{t}}}:\left(\mathbf{b}_{\mathbf{t}}: \dot{\boldsymbol{\eta}}-\mathbf{B}_{\mathbf{t}}^{\mathbf{e}}: \dot{\mathbf{N}}^{\mathrm{e}}\right)\right\rangle$

then:

$\dot{N}^{e}=M_{t}^{e}:\left(\left\langle b_{t}: A^{B_{t}}: \Gamma_{l}^{B_{t}}\right\rangle-I\right)^{-1}:\left\langle b_{t}: A^{B_{t}}: \Gamma_{l}^{B_{t}}: b_{t}: \dot{\eta}-b_{t}: \dot{\eta}\right\rangle$ 


\section{Acknowledgments}

This work was supported by the French State through the program "Investment in the future" operated by the National Research Agency (ANR) and referenced by ANR-11-LABX-000801 (Laboratory of Excellence 'DAMAS': Design of Alloy Metals for low-mAss Structures). 


\section{References}

Aeby-Gautier, E., Denand, B., Teixeira, J., Dehmas, M., Appolaire, B., Settefrati, A., 2011. Influence of microstructure on tensile properties of beta-metastable Ti-17 alloy. 12th World Conf Titan Beijing.

Ahston, P.J., Jun, T.S., Zhang, Z., Britton, T.B., Harte, A.M., Leen, S.B., Dunne, F.P.E., 2017. The effect of the beta phase on the micromechanical response of dual-phase titanium alloys. Int. J. Fatigue 100, 377-387.

Amouzou, K.E.K., Richeton, T., Roth, A., Lebyodkin, M.A., Lebedkina, T.A., 2016. Micromechanical modeling of hardening mechanisms in commercially pure $\alpha$-titanium in tensile condition. Int. J. Plast. 80, 222-240. https://doi.org/10.1016/j.ijplas.2015.09.008

Anahid, M., Samal, M. K., Ghosh, S., 2011. Dwell fatigue crack nucleation model based on crystal plasticity finite element simulations of polycrystalline titanium alloys. J. Mech. Phys. Solids 59, 2157-2176.

Armstrong, P.J., Frederick, C.O., 1966. A mathematical representation of the multiaxial Bauschinger effect. Report RD/B/N731, CEGB, Central Electricity Generating Board, Berkeley, UK.

Badr, O. M., Barlat, F., Rolfe, B., Lee, M. G., Hodgson, P., Weiss, M., 2016. Constitutive modelling of high strength titanium alloy Ti-6Al-4V for sheet forming applications at room temperature. Int. J. Solids Struct. 80, 334-347. http:// dx.doi.org/ 10.1016/ j. ijsolstr. 2015.08.025

Beal, J. D., Boyer, R., Sanders, D., 2006. Forming of Titanium and Titanium Alloys. In: ASM Handbook, Vol. 14B: Metalworking: Sheet Forming (S.L. Semiatin, ed.), p 656-669. DOI: 10.1361/asmhba0005146

Beausir, B., Fundenberger, J.-J., 2018. ATEX-software, Analysis Tools for Electron and X-ray diffraction, Université de Lorraine, <http://atex-software.eu/>.

Berbenni, S., Capolungo, L., 2015. A Mori-Tanaka homogenization scheme for non-linear elastoviscoplastic heterogeneous materials based on translated fields: An affine extension.

Comptes Rendus Mécanique 343, 95-106. http://dx.doi.org/10.1016/j.crme.2014.12.003

Berbenni, S., Favier, V., Berveiller, M., 2007. Impact of the grain size distribution on the yield stress of heterogeneous materials. Int. J. Plast. 23, 114-142. https://doi.org/10.1016/j.ijplas.2006.03.004

Berbenni, S., Favier, V., Lemoine, X., Berveiller, M., 2004. Micromechanical modeling of the elastic-viscoplastic behavior of polycrystalline steels having different microstructures. Mater. Sci. Eng. A 372, 128-136. https://doi.org/10.1016/j.msea.2003.11.010

Bunge, H.J., 1982. Texture Analysis in Materials Science. Academic-Verlag.

Cailletaud, G., Coudon, F., 2016. Scale Transition Rules Applied to Crystal Plasticity, in: Trovalusci, P. (Ed.), Materials with Internal Structure: Multiscale and Multifield Modeling and Simulation. Springer International Publishing, Cham, pp. 1-15. https://doi.org/10.1007/9783-319-21494-8 1

Cailletaud, G., 1992. A micromechanical approach to inelastic behaviour of metals. Int. J. Plast. 8, 55-73.

Cazacu, O., Plunkett, B., Barlat, F., 2006. Orthotropic yield criterion for hexagonal closed packed metals. Int. J. Plast. 22, 1171-1194.

Chaboche, J.L., Kanouté, P., Azzouz, F., 2012. Cyclic inelastic constitutive equations and their impact on the fatigue life predictions. Int. J. Plast. 35, 44-66. 
Chaussy, F., Driver, J.H., 1996. Evolutions microstructurales en compression uniaxiale à chaud de l'alliage $\beta$-Cez. Rev Met Paris 93, 1057-1066. https://doi.org/10.1051/metal/199693091057

Chini, M.R., Germain, L., Gey, N., Andrieu, S., Duval, T., 2016a. Advanced Microtexture Analysis of A Ti 10-2-3 Product for Better Understanding of Local Variations in Mechanical Behavior, in: Proceedings of the 13th World Conference on Titanium. John Wiley \& Sons, Inc., pp. 1943-1948.

Chini, M.R., Germain, L., Gey, N. 2016b. Private communication.

Clément, N., 2010. Phase transformations and mechanical properties of the Ti-5553 betametastable titanium alloy (PhD Thesis). UCL - FSA/MAPR - Département des sciences des matériaux et des procédés.

Clément, N., Lenain, A., Jacques, P.J., 2007. Mechanical property optimization via microstructural control of new metastable beta titanium alloys. JOM 59, 50-53. https://doi.org/10.1007/s11837-007-0010-y

DeWit, R., 2008. elastic constants and thermal expansion averages of a non textured polycrystals. J. Mech. Mater. Struct. 195-212.

Duval, T., 2013. Analyse multi-échelles des relations microstructure/propriétés mécaniques sous sollicitation monotone et cyclique des alliages de titane $\beta$-métastable (PhD Thesis). Ecole Nationale Supérieure de Mécanique et d'Aérotechnique.

Duval, T., Villechaise, P., Andrieu, S., The Minerals, M.\& M.S. (TMS), 2011. Mechanical Properties and Strain Mechanisms Analysis in Ti-5553 Titanium Alloy, in: Supplemental Proceedings. John Wiley \& Sons, Inc., pp. 471-478. https://doi.org/10.1002/9781118062173.ch59

Eshelby, J.D., 1957. The Determination of the Elastic Field of an Ellipsoidal Inclusion, and Related Problems. Proc. R. Soc. Lond. Math. Phys. Eng. Sci. 241, 376-396. https://doi.org/10.1098/rspa.1957.0133

Fisher, E.S., Dever, D., 1970. Relation of the c' elastic modulus to stability of b.c.c. transition metals. Acta Metall. 18, 265-269. http://dx.doi.org/10.1016/0001-6160(70)90033-7

Fréour, S., Gloaguen, D., François, M., Perronnet, A., Guillén, R., 2005. Determination of singlecrystal elasticity constants in a cubic phase within a multiphase alloy: X-ray diffraction measurements and inverse-scale transition modelling. J. Appl. Crystallogr. 38, 30-37. https://doi.org/10.1107/S0021889804023441

Fréour, S., Lacoste, E., François, M., Guillén, R., 2011. Determining Ti-17 $\beta$-phase single-crystal elasticity constants through $X$-ray diffraction and inverse scale transition model, in: Materials Science Forum. Trans Tech Publ, pp. 97-102.

Ghosh, S., Anahid, M., 2013. Homogenized constitutive and fatigue nucleation models from crystal plasticity FE simulations of Ti alloys, Part 1: Macroscopic anisotropic yield function. Int. J. Plast. 47, 182-201.

Hershey, A.V., 1954. The elasticity of an isotropic aggregate of anisotropic cubic crystals. J. Appl. Mech.-Trans. ASME 21, 236-240.

Hill, R., 1965. Theory of mechanical properties of fibre-strengthened materials-III. self-consistent model. J. Mech. Phys. Solids 13, 189-198.

Hounkpati, V., Fréour, S., Gloaguen, D., Legrand, V., Kelleher, J., Kockelmann, W., Kabra, S., 2016. In situ neutron measurements and modelling of the intergranular strains in the nearbeta titanium alloy Ti- $\beta$ 21S. Acta Mater. 109, 341-352.

Huang, S., Brown, D. W., Clausen, B., Teng, Z., Gao, Y., Liaw, P. K., 2012. In situ neutron diffraction studies on the creep behavior of a ferritic superalloy. Metall. Mater. Trans. 43A, 1497-1508. 
Huang, S., Gao, Y., An K., Zheng, L., Wu, W., Teng, Z., Liaw, P. K., 2015. Deformation mechanisms in a precipitation-strengthened ferritic superalloy revealed by in situ neutron diffraction studies at elevated temperatures. Acta Mater. 83 , 1497-1508.

Khan, A.S., Huang, S., 1995. Continuum theory of plasticity. John Wiley \& Sons, New York.

Khan, A.S., Yu, S., Liu, H., 2012. Deformation induced anisotropic responses of Ti-6Al-4V alloy Part II: A strain rate and temperature dependent anisotropic yield criterion. Int. J. Plast. 38, 14-26.

Kim, J.-Y., Rokhlin, S.I., 2009. Determination of elastic constants of generally anisotropic inclined lamellar structure using line-focus acoustic microscopy. J. Acoust. Soc. Am. 126, 29983007. https://doi.org/10.1121/1.3245032

Kröner, E., 1990. Modified Green Functions in the Theory of Heterogeneous and/or Anisotropic Linearly Elastic Media, in: Weng, G.J., Taya, M., Abé, H. (Eds.), Micromechanics and Inhomogeneity: The Toshio Mura 65th Anniversary Volume. Springer New York, New York, NY, pp. 197-211. https://doi.org/10.1007/978-1-4613-8919-4_13

Kröner, E., 1958. Computation of the elastic constants of polycrystals from constants of single crystals. Z Phys 151, 504-518.

Lahellec, N., Suquet, P., 2007. On the effective behavior of nonlinear inelastic composites: I. Incremental variational principles. J. Mech. Phys. Solids 55, 1932-1963. https://doi.org/10.1016/j.jmps.2007.02.003

Ledbetter, H., Ogi, H., Kai, S., Kim, S., Hirao, M., 2004. Elastic constants of body-centered-cubic titanium monocrystals. J. Appl. Phys. 95, 4642-4644. https://doi.org/10.1063/1.1688445

Lemoine, X., Aouafi,A., 2008. Bauschinger effect correspondence of experimental tests. Int. J. Mater. Form. 1 (1), 241-244.

Liu, Y., Gilormini, P., Castañeda, P.P., 2003. Variational self-consistent estimates for texture evolution in viscoplastic polycrystals. Acta Mater. 51, 5425-5437. https://doi.org/10.1016/S1359-6454(03)00409-9

Mandal, S., Gockel, B.T., Balachandran, S., Banerjee, D., Rollett, A.D., 2017. Simulation of plastic deformation in Ti-5553 alloy using a self-consistent viscoplastic model. Int. J. Plast. 94, 5773.

Mareau, C., Berbenni, S., 2015. An affine formulation for the self-consistent modeling of elastoviscoplastic heterogeneous materials based on the translated field method. Int. J. Plast. 64, 134-150. http://dx.doi.org/10.1016/j.ijplas.2014.08.011

Martin, G., 2012. Simulation numérique multi-échelles du comportement mécanique des alliages de titane bêta-métastable Ti5553 et Ti17 (PhD Thesis). Ecole nationale supérieure des Mines de Paris.

Martin, G., Naźe, L., Cailletaud, G., 2011. Numerical multi-scale simulations of the mechanical behavior of $\beta$-metastable titanium alloys Ti5553 and Ti17. Procedia Eng. 10, 1803-1808. https://doi.org/10.1016/j.proeng.2011.04.300

Masson, R., Bornert, M., Suquet, P., Zaoui, A., 2000. An affine formulation for the prediction of the effective properties of nonlinear composites and polycrystals. J. Mech. Phys. Solids 48, 1203-1227. https://doi.org/10.1016/S0022-5096(99)00071-X

Masson, R., Zaoui, A., 1999. Self-consistent estimates for the rate-dependentelastoplastic behaviour of polycrystalline materials. J. Mech. Phys. Solids 47, 1543-1568. https://doi.org/10.1016/S0022-5096(98)00106-9

Mercier, S., Molinari, A., 2009. Homogenization of elastic-viscoplastic heterogeneous materials: Self-consistent and Mori-Tanaka schemes. Int. J. Plast. 25, 1024-1048. https://doi.org/10.1016/j.jplas.2008.08.006 
Mercier, S., Molinari, A., Berbenni, S., Berveiller, M., 2012. Comparison of different homogenization approaches for elastic-viscoplastic materials. Model. Simul. Mater. Sci. Eng. 20, 024004.

Meredith, C. S., Khan, A.S., 2012. Texture evolution and anisotropy in the thermo-mechanical response of UFG Ti processed via equal channel angular pressing. Int. J. Plast. 30-31, 202217.

Méric, L., Cailletaud, G., 1991. Single Crystal Modeling for Structural Calculations: Part 2-Finite Element Implementation. J. Eng. Mater. Technol. 113, 171-182. https://doi.org/10.1115/1.2903375

Méric, L., Poubanne, P., Cailletaud, G., 1991. Single Crystal Modeling for Structural Calculations: Part 1-Model Presentation. J. Eng. Mater. Technol. 113, 162-170. https://doi.org/10.1115/1.2903374

Molinari, A., 2001. Averaging Models for Heterogeneous Viscoplastic and Elastic Viscoplastic Materials. J. Eng. Mater. Technol. 124, 62-70. https://doi.org/10.1115/1.1421052

Nejezchlebová, J., Janovská, M., Seiner, H., Sedlák, P., Landa, M., Šmilauerová, J., Stráský, J., Harcuba, P., Janeček, M., 2016. The effect of athermal and isothermal $\omega$ phase particles on elasticity of $\beta-\mathrm{Ti}$ single crystals. Acta Mater. 110, 185-191. http://dx.doi.org/10.1016/j.actamat.2016.03.033

Nicaise, N., Berbenni, S., Wagner, F., Berveiller, M., Lemoine, X., 2011. Coupled effects of grain size distributions and crystallographic textures on the plastic behaviour of IF steels. Int. J. Plast. 27, 232-249. https://doi.org/10.1016/j.ijplas.2010.05.001

Nyakana, S.L., Fanning, J.C., Boyer, R.R., 2005. Quick reference guide for $\beta$ titanium alloys in the 00s. J. Mater. Eng. Perform. 14, 799-811. https://doi.org/10.1361/105994905X75646

Paquin, A., Berbenni, S., Favier, V., Lemoine, X., Berveiller, M., 2001. Micromechanical modeling of the elastic-viscoplastic behavior of polycrystalline steels. Int. J. Plast. 17, 1267-1302. https://doi.org/10.1016/S0749-6419(00)00047-4

Paquin, A., Sabar, H., Berveiller, M., 1999. Integral formulation and self-consistent modelling of elastoviscoplastic behavior of heterogeneous materials. Arch. Appl. Mech. 69, 14-35. https://doi.org/10.1007/s004190050201

Petry, W., Heiming, A., Trampenau, J., Alba, M., Herzig, C., Schober, H.R., Vogl, G., 1991. Phonon dispersion of the bcc phase of group-IV metals. I. bcc titanium. Phys Rev B 43, 10933-10947. https://doi.org/10.1103/PhysRevB.43.10933

Raabe, D., Sander, B., Friak, M., Neugebauer, J., 2008. Ab-initio simulation and experimental validation of beta-titanium alloys. Eprint ArXiv08110157.

Raghunathan, S.L., Stapleton, A.M., Dashwood, R.J., Jackson, M., Dye, D., 2007. Micromechanics of Ti-10V-2Fe-3Al: In situ synchrotron characterisation and modelling. Acta Mater. 55, 6861-6872. http://dx.doi.org/10.1016/j.actamat.2007.08.049

Reid, C.N., Routbort, J.L., Maynard, R.A., 1973. Elastic constants of Ti-40 at.\% Nb at $298{ }^{\circ} \mathrm{K} . \mathrm{J}$. Appl. Phys. 44, 1398-1399. https://doi.org/10.1063/1.1662365

Sabar, H., Berveiller, M., Favier, V., Berbenni, S., 2002. A new class of micro-macro models for elastic-viscoplastic heterogeneous materials. Int. J. Solids Struct. 39, 3257-3276. https://doi.org/10.1016/S0020-7683(02)00256-1

Sauzay, M., 2006. Effet de l'anisotropie élastique cristalline sur la distribution des facteurs de Schmid à la surface des polycristaux. Comptes Rendus Mécanique 334, 353-361. https://doi.org/10.1016/j.crme.2006.03.004

Settefrati, A., 2012. Étude expérimentale et modélisation par champ de phase de la formation de alpha dans les alliages de titane bêta-métastable (PhD Thesis). Université de Lorraine.

Smith, B.D., Shih, D.S., McDowell, D.L., 2018. Cyclic plasticity experiments and polycrystal plasticity modeling of three distinct Ti alloy microstructures. Int. J. Plast. 101, 1-23. 
Sowerby, R., Uko, D.K., Tomita, Y., 1979. A review of certain aspects of the Bauschinger effect in metals. Mater. Sci. Eng. 41, 43-58.

Tane, M., Akita, S., Nakano, T., Hagihara, K., Umakoshi, Y., Niinomi, M., Nakajima, H., 2008. Peculiar elastic behavior of $\mathrm{Ti}-\mathrm{Nb}-\mathrm{Ta}-\mathrm{Zr}$ single crystals. Acta Mater. 56, 2856-2863. http://dx.doi.org/10.1016/j.actamat.2008.02.017

Taupin, V., Pesci, R., Berbenni, S., Berveiller, S., Ouahab, R., Bouaziz, O., 2013. Lattice strain measurements using synchrotron diffraction to calibrate a micromechanical modeling in a ferrite-cementite steel. Mater. Sci. Eng. A 561, 67-77. https://doi.org/10.1016/j.msea.2012.10.086

Turner, P.A., Tomé, C.N., 1994. A study of residual stresses in Zircaloy-2 with rod texture. Acta Metall. Mater. 42, 4143-4153. https://doi.org/10.1016/0956-7151(94)90191-0

Wang, H., Wu, P.D., Tomé, C.N., Huang, Y., 2010. A finite strain elastic-viscoplastic selfconsistent model for polycrystalline materials. J. Mech. Phys. Solids 58, 594-612. https://doi.org/10.1016/j.jmps.2010.01.004

Wang, H., Wu, P.D., Wang, J., Tomé, C.N., 2013. A crystal plasticity model for hexagonal close packed (HCP) crystals including twinning and de-twinning mechanisms. Int. J. Plast. 49, 3652. 